\title{
18. X-RAY MINERALOGY DATA FROM THE NORTHWEST PACIFIC, LEG 32, DEEP SEA DRILLING PROJECT ${ }^{1}$
}

\author{
I. Zemmels and H.E. Cook, University of California, Riverside, California
}

\section{METHODS}

Semiquantitative determinations of the mineral composition of bulk samples, $2-20 \mu \mathrm{m}$, and $<2 \mu \mathrm{m}$ fractions were performed according to the methods described in the appendix of Volume 28, Initial Reports of the Deep Sea Drilling Project.

The method of sample preparation, in brief, is as follows: Bulk samples are washed to remove seawater salts and are ground to less than $10 \mu \mathrm{m}$ under butanol. A portion of the sediment is decalcified in a sodium-

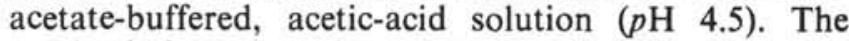
residue is fractionated into $2-20 \mu \mathrm{m}$ and $<2 \mu \mathrm{m}$ samples by wet-sieving and centrifugation. The $2-20 \mu \mathrm{m}$ samples are ground to less than $10 \mu \mathrm{m}$. These three preparations are treated with trihexylamine acetate to expand the smectites. All samples are X-rayed as random powders.

The results of the X-ray diffraction analysis are presented in Tables 1 to 18 . Tables 1 to 9 summarize the mineral data provide stratigraphic information and sample identification. The sediment ages, lithologic units, and nomenclature of the sediment types are from the DSDP Leg 32 Hole Summaries and from a subsequent update supplied by Dr. James V. Gardner, DSDP.

The percentage of amorphous material is a measure of the weight fraction of amorphous material in each sample, which commonly consists of biogenic silica, volcanic glass, palagonite, allophane, and organic material. The amorphous content is calculated from the total diffuse scattering of the sample. The method of calculation assumes that the diffuse scatter in excess of the diffuse scatter from the crystalline materials is

'Institute of Geophysics and Planetary Physics, University of California, Riverside, California, Contribution No. 74-27. proportioned to the amorphous content. The diffuse scatter of the crystalline minerals is determined from the mineral calibration standards (see Appendix, Volume 28 ). Ideally, the amorphous content varies between zero and $100 \%$, but, in cases where the minerals in the sample have a higher degree of crystallinity than the calibration standards, negative values can result. The negative values are reported as blanks and these samples can be assumed to contain little or no amorphous material.

The crystalline minerals are quantified by the method of mutual ratios using peak heights and concentration factors derived from ratioing the diagnostic peaks of minerals with the major peak of quartz. Unquantifiable minerals, i.e., unidentified minerals and minerals for which standards are not available, are tentatively quantified using a hypothetical concentration factor of 3.0 which is applied to the major peak of the mineral. The concentrations of the quantifiable minerals is summed to $100 \%$. The amorphous content and the unquantifiable minerals are not included in the total. The unquantifiable minerals are reported on a qualitative scale as trace (less than $5 \%)$, present $(5 \%-25 \%)$, abundant $(25 \%$ $65 \%$ ) and major (greater than $65 \%$ ).

The precision of the mineral determination is approximately \pm 1 weight percent of the amount present. Because of differences in the crystallinity between the mineral calibration standards and the minerals in the samples and also diffraction peak interferences, the accuracy of the reported concentrations is often less than the precision of the method allows. In terms of the reported concentration, smectites may vary $\pm 50 \%$; micas, chlorites, cristobalite, tridymite, and goethite may vary $\pm 20 \%$; kaolinite, amphibole, augite, the feldspars, the zeolites, palygorskite, sepiolite and apatite may vary $\pm 10 \%$; the minerals which have stable crystal lattices and are not members of solid-solution series (or typically have limited crystal-lattice substitution in the sedimentary environment) such as quartz, low-

TABLE 1

Summary of X-Ray Mineralogy Samples, Sample Depths, Lithology, Age, and X-Ray Diffraction Results, Site 303

\begin{tabular}{|c|c|c|c|c|c|c|c|c|c|c|c|c|}
\hline \multirow{2}{*}{$\begin{array}{c}\text { Sample } \\
\text { (Interval in } \mathrm{cm} \text { ) }\end{array}$} & \multirow{2}{*}{$\begin{array}{l}\text { Sample Depth } \\
\text { Below Sea } \\
\text { Floor (m) }\end{array}$} & \multirow[b]{2}{*}{ Lithology } & \multirow[b]{2}{*}{ Age } & \multicolumn{3}{|c|}{$\begin{array}{c}\text { Bulk Sample } \\
\text { Major Constituent }\end{array}$} & \multicolumn{3}{|c|}{$\begin{array}{l}2-20 \mu \mathrm{m} \text { Fraction } \\
\text { Major Constituent }\end{array}$} & \multicolumn{3}{|c|}{$\begin{array}{l}<2 \mu \mathrm{m} \text { Fraction } \\
\text { Major Constituent }\end{array}$} \\
\hline & & & & $T$ & 2 & 3 & 1 & 2 & 3 & 1 & 2 & 3 \\
\hline Hole 303 & & & & & & & & & & & & \\
\hline $\begin{array}{l}2-3,20 \\
3-5,136 \\
4-2,80\end{array}$ & $\begin{array}{r}65.2 \\
124.4 \\
176.3\end{array}$ & $\begin{array}{c}\text { Unit } 1 \\
\text { Rad Diatom ooze } \\
\text { and pelagic clay }\end{array}$ & $\begin{array}{c}\text { Early Plio. } \\
\text { to late } \\
\text { Miocene }\end{array}$ & $\begin{array}{l}\text { Quar. } \\
\text { Quar. } \\
\text { No }\end{array}$ & $\begin{array}{l}\text { Plag. } \\
\text { Mica } \\
\text { ata }\end{array}$ & $\begin{array}{l}\text { Mica } \\
\text { Plag. }\end{array}$ & $\begin{array}{l}\text { Quar. } \\
\text { Quar. } \\
\text { Quar. }\end{array}$ & $\begin{array}{l}\text { Mica } \\
\text { Mica } \\
\text { Mica }\end{array}$ & $\begin{array}{l}\text { Plag. } \\
\text { Plag. } \\
\text { Plag. }\end{array}$ & $\begin{array}{l}\text { Mont. } \\
\text { Mont. } \\
\text { Mica }\end{array}$ & $\begin{array}{l}\text { Mica } \\
\text { Mica } \\
\text { Quar. }\end{array}$ & $\begin{array}{l}\text { Quar. } \\
\text { Quar. } \\
\text { Mont. }\end{array}$ \\
\hline Hole $303 \mathrm{~A}$ & & & & & & & & & & & & \\
\hline $5-2,118$ & 250.5 & Unit $2^{\mathrm{a}}$ & a & Mont. & Clin. & Quar. & Clin. & Mont. & Plag. & Mont. & $\mathrm{K}$-Fe. & \\
\hline
\end{tabular}

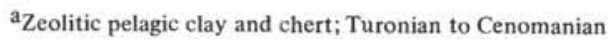


magnesium calcite, aragonite, dolomite, rhodochrosite, siderite, gibbsite, talc, barite, anatase, gypsum, anhydrite, halite, pyrite, hematite, and magnetite will vary less than $\pm 5 \%$.

The user of the X-ray mineralogy data should bear in mind that (1) the reported values are not absolute concentrations and some adjustment has to be made for the amorphous content and the unquantifiable minerals; (2) in a homogeneous system of minerals, the mineral concentration trends are reliable because of the precision, but when comparing mineral concentrations between different geographic regions or lithologic units, additional information regarding the crystallinity of the minerals is required; (3) the representativeness of the samples selected for X-ray diffraction analysis is the responsibility of the shipboard scientists and any questions pertaining to this aspect should be directed to them.

\section{DRILLING MUD USAGE}

Drilling mud, containing montmorillonite and barite, was used in Hole 303A between Cores 7 and 8, Cores 9 and 10; Hole 305 between Cores 43 and 44; Hole 307 between Cores 9 and 10; and Hole 310A while cutting Core 16 and between Cores 17 and 18. None of the samples submitted for X-ray diffraction analysis was directly exposed to the drilling mud.

\section{ACKNOWLEDGMENTS}

The writers wish to acknowledge the excellent work of Nicki D. Coursey in the interpretation of X-ray diffraction data, of Paul D. Johnson in X-ray data acquisition and data processing, and of Tom W. Halverson, Jr., in sample preparation.

TABLE 2

Summary of X-Ray Mineralogy Samples, Sample Depths, Lithology, Age, and X-Ray Diffraction Results, Site 304

\begin{tabular}{|c|c|c|c|c|c|c|c|c|c|c|c|c|}
\hline \multirow{2}{*}{$\begin{array}{c}\text { Sample } \\
\text { (Interval in } \mathrm{cm} \text { ) }\end{array}$} & \multirow{2}{*}{$\begin{array}{l}\text { Sample Depth } \\
\text { Below Sea } \\
\text { Floor }(\mathrm{m})\end{array}$} & \multirow[b]{2}{*}{ Lithology } & \multirow[b]{2}{*}{ Age } & \multicolumn{3}{|c|}{$\begin{array}{c}\text { Bulk Sample } \\
\text { Major Constituent }\end{array}$} & \multicolumn{3}{|c|}{$\begin{array}{l}2-20 \mu \mathrm{m} \text { Fraction } \\
\text { Major Constituent }\end{array}$} & \multicolumn{3}{|c|}{$\begin{array}{l}<2 \mu \mathrm{m} \text { Fraction } \\
\text { Major Constituent }\end{array}$} \\
\hline & & & & 1 & 2 & 3 & 1 & 2 & 3 & 1 & 2 & 3 \\
\hline $\begin{array}{l}1-4,8 \\
2-2,92\end{array}$ & $\begin{array}{l}110.1 \\
218.4\end{array}$ & $\begin{array}{l}\text { Unit } 1^{\mathrm{a}} \\
\text { Unit } 2^{\mathrm{b}}\end{array}$ & $\begin{array}{l}\mathrm{a} \\
\mathrm{b}\end{array}$ & $\begin{array}{l}\text { Quar. } \\
\text { Mont. }\end{array}$ & $\begin{array}{l}\text { Mica } \\
\text { Quar. }\end{array}$ & $\begin{array}{l}\text { Plag. } \\
\text { Mica }\end{array}$ & $\begin{array}{l}\text { Quar. } \\
\text { Quar. }\end{array}$ & $\begin{array}{l}\text { Mica } \\
\text { Mica }\end{array}$ & $\begin{array}{l}\text { Plag. } \\
\text { Plag. }\end{array}$ & $\begin{array}{l}\text { Mica } \\
\text { Mont. }\end{array}$ & $\begin{array}{l}\text { Mont. } \\
\text { Mica }\end{array}$ & $\begin{array}{l}\text { Quar. } \\
\text { Quar. }\end{array}$ \\
\hline
\end{tabular}

a Radiolarian diatom ooze; late Miocene

${ }^{\mathrm{b}}$ Unfossiliferous pelagic clay; Miocene cavings.

TABLE 3

Summary of X-Ray Mineralogy Samples, Sample Depths, Lithology, Age, and X-Ray Diffraction Results, Site 305

\begin{tabular}{|c|c|c|c|c|c|c|c|c|c|c|c|c|}
\hline \multirow{2}{*}{$\begin{array}{c}\text { Sample } \\
\text { (Interval in } \mathrm{cm} \text { ) }\end{array}$} & \multirow{2}{*}{$\begin{array}{l}\text { Sample Depth } \\
\text { Below Sea } \\
\text { Floor (m) }\end{array}$} & \multirow[b]{2}{*}{ Lithology } & \multirow[b]{2}{*}{ Age } & \multicolumn{3}{|c|}{$\begin{array}{c}\text { Bulk Sample } \\
\text { Major Constituent }\end{array}$} & \multicolumn{3}{|c|}{$\begin{array}{l}2-20 \mu \mathrm{m} \text { Fraction } \\
\text { Major Constituent }\end{array}$} & \multicolumn{3}{|c|}{$\begin{array}{l}<2 \mu \mathrm{m} \text { Fraction } \\
\text { Major Constituent }\end{array}$} \\
\hline & & & & 1 & 2 & 3 & 1 & 2 & 3 & 1 & 2 & 3 \\
\hline $\begin{array}{l}4-3,43 \\
5-2,130\end{array}$ & $\begin{array}{l}29.9 \\
38.3\end{array}$ & \multirow{2}{*}{$\begin{array}{c}\text { Unit 1 } \\
\begin{array}{c}\text { Foram-bearing } \\
\text { nanno ooze }\end{array} \\
\end{array}$} & \multirow{2}{*}{$\begin{array}{c}\text { Pleistocene } \\
\text { to } \\
\text { Pliocene }\end{array}$} & \multirow{2}{*}{$\begin{array}{l}\text { Calc. } \\
\text { Calc. } \\
\text { Calc. }\end{array}$} & \multirow[b]{2}{*}{ Mica } & \multirow[b]{2}{*}{ Quar. } & \multirow{2}{*}{$\begin{array}{l}\text { Quar. } \\
\text { Quar. } \\
\text { Mica }\end{array}$} & \multirow{2}{*}{$\begin{array}{l}\text { Mica } \\
\text { Mica } \\
\text { Quar. }\end{array}$} & \multirow{2}{*}{$\begin{array}{l}\text { Plag. } \\
\text { Plag. } \\
\text { Plag. }\end{array}$} & \multicolumn{3}{|c|}{$\begin{array}{l}\text { Mica }\left.\right|_{\text {Insufficient residue }} \text { Quar. } \\
\text { Mont }\end{array}$} \\
\hline $5-4,83$ & 40.8 & & & & & & & & & Mica. & Quar. & Mont. \\
\hline $6-4,86$ & 50.4 & \multirow{13}{*}{$\begin{array}{c}\text { Unit } 2 \\
\text { Foram-bearing } \\
\text { nanno ooze }\end{array}$} & \multirow{13}{*}{$\begin{array}{l}\text { Maestrich- } \\
\text { tian to late } \\
\text { Miocene }\end{array}$} & Calc. & & & Phil. & Quar. & Mica & Mica & Quar. & Mont. \\
\hline $6-5,20$ & 51.2 & & & Calc. & & & Mica & Phil. & Quar. & Mica & Mont. & Quar. \\
\hline $6-5,130$ & 52.3 & & & Calc. & & & Phil. & Quar. & Mica & Paly. & Quar. & Phil. \\
\hline $7-2,102$ & 57.0 & & & Calc. & & & Phil. & Quar. & $\mathrm{K}-\mathrm{Fe}$. & Mica & Quar. & Paly. \\
\hline $7-5,50$ & 61.0 & & & Calc. & & & Phil. & Quar. & Plag. & Mica & Quar. & $\mathrm{K}-\mathrm{Fe}$. \\
\hline $8-2,100$ & 66.5 & & & Calc. & & & \multicolumn{3}{|c|}{ Insufficient residue } & Mica & Quar. & Paly. \\
\hline $9-5,100$ & 80.0 & & & Calc. & & & Quar. & $\mathrm{K}-\mathrm{Fe}$. & Phil. & Mica & Paly. & Quar. \\
\hline $10-5,100$ & 89.0 & & & Calc. & & & Quar. & $\mathrm{K}$-Fe. & Plag. & Mica & Quar. & K-Fe. \\
\hline $11-2,66$ & 93.7 & & & Calc. & & & K-Fe. & Plag. & Quar. & Mica & Paly. & Quar. \\
\hline $13-2,100$ & 113.0 & & & Calc. & & & $\mathrm{K}-\mathrm{Fe}$. & Quar. & & Mont. & & \\
\hline $15-5,99$ & 136.5 & & & Calc. & & & $\mathrm{K}-\mathrm{Fe}$. & Quar. & Mica & Mont. & Paly. & Mica \\
\hline $16-2,100$ & 141.5 & & & Calc. & & & \multirow{2}{*}{\multicolumn{3}{|c|}{$\begin{array}{l}\text { Insufficient residue } \\
\text { Insufficient residue }\end{array}$}} & Mont. & Mica & Quar. \\
\hline $16-5,3$ & 145.0 & & & Calc. & & & & & & Mica & Mont. & Quar. \\
\hline $17-5,100$ & 155.5 & Unit 3 & Companian & Calc. & & & \multirow{4}{*}{\multicolumn{3}{|c|}{$\begin{array}{l}\text { Insufficient residue } \\
\text { Insufficient residue } \\
\text { Insufficient residue } \\
\text { Insufficient residue }\end{array}$}} & Mont. & Paly. & Mica \\
\hline $20-5,102$ & 183.5 & Foram nanno & to Maestrich- & Calc. & & & & & & Mont. & Mica & Paly. \\
\hline $23-5,102$ & 212.0 & ooze, chalk & tian & Calc. & & & & & & Mont. & Paly. & Quar. \\
\hline $25-5,99$ & 230.5 & and chert & & Calc. & & & & & & Ins & ficient $\mathrm{re}$ & due \\
\hline $\begin{array}{l}59-1,140 \\
59-1,130\end{array}$ & $\begin{array}{l}551.9 \\
551.8\end{array}$ & \multirow{5}{*}{$\begin{array}{l}\text { Unit } 4 \\
\text { limestone } \\
\text { porcellanite } \\
\text { and chert }\end{array}$} & \multirow{5}{*}{$\begin{array}{l}\text { Aptian } \\
\text { to } \\
\text { Barremian }\end{array}$} & $\begin{array}{l}\text { Calc. } \\
\text { Quar. }\end{array}$ & $\begin{array}{l}\text { Quar. } \\
\text { Calc. }\end{array}$ & \multirow{5}{*}{ Trid. } & \multicolumn{2}{|c|}{ Insufficient residue } & $\begin{array}{l}\text { Bari. } \\
\text { idue }\end{array}$ & \multicolumn{3}{|c|}{ Insufficient residue } \\
\hline $65-1,100$ & 608.0 & & & Quar. & Cris. & & Quar. & Cris. & Pyri. & Mont. & Cris. & \\
\hline $65-1,129$ & 608.3 & & & Cris. & Quar. & & Cris. & Quar. & Trid. & Cris. & Trid. & Quar. \\
\hline $66-1,115$ & 617.7 & & & Calc. & Quar. & & Quar. & (n) & & Quar. & Paly. & Mica \\
\hline $60-1,104$ & 561.0 & & & Quar. & Calc. & & \multicolumn{3}{|c|}{ Insufficient residue } & \multicolumn{3}{|c|}{ Insufficient residue } \\
\hline
\end{tabular}


TABLE 4

Summary of X-Ray Mineralogy Samples, Sample Depths, Lithology, Age, and X-Ray Diffraction Results, Site 306

\begin{tabular}{|c|c|c|c|c|c|c|c|c|c|c|c|c|}
\hline \multirow{2}{*}{$\begin{array}{c}\text { Sample } \\
\text { (Interval in } \mathrm{cm} \text { ) }\end{array}$} & \multirow{2}{*}{$\begin{array}{l}\text { Sample Depth } \\
\text { Below Sea } \\
\text { Floor (m) }\end{array}$} & \multirow[b]{2}{*}{ Lithology } & \multirow[b]{2}{*}{ Age } & \multicolumn{3}{|c|}{$\begin{array}{c}\text { Bulk Sample } \\
\text { Major Constituent }\end{array}$} & \multicolumn{3}{|c|}{$\begin{array}{l}2-20 \mu \mathrm{m} \text { Fraction } \\
\text { Major Constituent }\end{array}$} & \multicolumn{3}{|c|}{$\begin{array}{c}<2 \mu \mathrm{m} \text { Fraction } \\
\text { Major Constituent }\end{array}$} \\
\hline & & & & 1 & 2 & 3 & 1 & 2 & 3 & 1 & 2 & 3 \\
\hline $1-2,128$ & 2.8 & Unit $1^{\mathrm{a}}$ & a & Calc. & Quar. & & Quar. & Mica & Plag. & Mica & Quar. & \\
\hline $8-1,104$ & 114.0 & Unit $3 b$ & b & Calc. & Quar. & Trid. & Cris. & Quar. & Trid. & Trid. & Cris. & \\
\hline $\begin{array}{l}21-1,67 \\
21-1,144 \\
29-7,0 \\
36-7,0 \\
40-1,135\end{array}$ & $\begin{array}{l}281.7 \\
282.4 \\
364.5 \\
420.5 \\
450.9\end{array}$ & $\begin{array}{c}\text { Unit c } \\
\text { Nanno chalk } \\
\text { and chert }\end{array}$ & c & $\begin{array}{l}\text { Calc. } \\
\text { Calc. } \\
\text { Calc. } \\
\text { Calc. } \\
\text { Calc. }\end{array}$ & $\begin{array}{l}\text { Quar. } \\
\text { Quar. } \\
\text { Quar. }\end{array}$ & & $\begin{array}{l}\text { Quar. } \\
\text { Quar. } \\
\text { Quar. } \\
\text { Bari. } \\
\text { Bari. }\end{array}$ & $\begin{array}{l}\text { Mica } \\
\text { Mica } \\
\text { K-Fe. } \\
\text { Mica }\end{array}$ & $\begin{array}{l}\text { K-Fe. } \\
\text { K-Fe. } \\
\text { Pyri. } \\
\text { Quar. }\end{array}$ & $\begin{array}{l}\text { Quar. } \\
\text { Quar. } \\
\text { Quar. } \\
\text { Mixl. } \\
\text { Mixl. }\end{array}$ & $\begin{array}{l}\text { Mica } \\
\text { Mixl. } \\
\text { Mont. } \\
\text { Quar. }\end{array}$ & \\
\hline
\end{tabular}

${ }^{\mathrm{a}}$ Foram nanno ooze; mixed Quaternary and Albian.

${ }_{\text {Radiolarian-bearing porcellanite and chert; Aptian. }}$

$\mathrm{c}_{\text {Barremian-Hauterivian to Valanginian. }}$

TABLE 5

Summary of X-Ray Mineralogy Samples, Sample Depths, Lithology, Age, and X-Ray Diffraction Results, Site 307

\begin{tabular}{|c|c|c|c|c|c|c|c|c|c|c|c|c|}
\hline \multirow{2}{*}{$\begin{array}{c}\text { Sample } \\
\text { (Interval in } \mathrm{cm} \text { ) }\end{array}$} & \multirow{2}{*}{$\begin{array}{l}\text { Sample Depth } \\
\text { Below Sea } \\
\text { Floor }(m)\end{array}$} & \multirow[b]{2}{*}{ Lithology } & \multirow[b]{2}{*}{ Age } & \multicolumn{3}{|c|}{$\begin{array}{c}\text { Bulk Sample } \\
\text { Major Constituent }\end{array}$} & \multicolumn{3}{|c|}{$\begin{array}{l}2-20 \mu \mathrm{m} \text { Fraction } \\
\text { Major Constituent }\end{array}$} & \multicolumn{3}{|c|}{$\begin{array}{l}<2 \mu \mathrm{m} \text { Fraction } \\
\text { Major Constituent }\end{array}$} \\
\hline & & & & 1 & 2 & 3 & 1 & 2 & 3 & 1 & 2 & 3 \\
\hline $1-2,100$ & 2.5 & Unit $1^{a}$ & a & Mica & Quar. & Plag. & Mica & Quar. & Plag. & Mica & Mont. & Quar. \\
\hline $5-7,0$ & 112.0 & Unit $2 b$ & $\frac{a}{b}$ & Plag. & Mont. & Clin. & Plag. & Clin. & Mont. & Mont. & Plag. & $\mathrm{K}-\mathrm{Fe}$. \\
\hline $9-1,108$ & 233.6 & Unit 3 & 0 & Quar. & Dolo. & & \multicolumn{3}{|c|}{ Insufficient residue } & \multicolumn{3}{|c|}{ Insufficient residue } \\
\hline $\begin{array}{l}12-7,0 \\
11-1,100 \\
12-1\end{array}$ & $\begin{array}{l}306.5 \\
289.5 \\
298.6\end{array}$ & $\begin{array}{l}\text { Chert, nanno chalk } \\
\text { and calc. porcell. }\end{array}$ & $\begin{array}{l}\text { Early } \\
\text { Cret. }\end{array}$ & K-Fe. & $\begin{array}{l}\text { Mont. } \\
\text { Quar. }\end{array}$ & $\begin{array}{l}\text { Quar. } \\
\text { Cris. }\end{array}$ & $\begin{array}{l}\text { K-Fe. } \\
\text { Quar. }\end{array}$ & $\begin{array}{l}\text { Mont. } \\
\text { Kaol. }\end{array}$ & Quar. & Mont. & $\begin{array}{l}\text { K-Fe. } \\
\text { Kaol. }\end{array}$ & $\begin{array}{l}\text { Kaol. } \\
\text { Mica }\end{array}$ \\
\hline $12-1,108$ & 298.6 & & & Quar. & Mixl. & Hema. & & Hema. & & Quar. & Mixl. & Hema. \\
\hline
\end{tabular}

${ }^{\mathrm{a}}$ Zeolitic pelagic clay; Quaternary.

${ }^{6}$ Radiolarian-bearing altered ash; Albian.

TABLE 6

Summary of X-Ray Mineralogy Samples, Sample Depths, Lithology, Age, and X-Ray Diffraction Results, Site 308

\begin{tabular}{|c|c|c|c|c|c|c|c|c|c|c|c|c|}
\hline \multirow{2}{*}{$\begin{array}{c}\text { Sample } \\
(\text { Interval in } \mathrm{cm})\end{array}$} & \multirow{2}{*}{$\begin{array}{l}\text { Sample Depth } \\
\text { Below Sea } \\
\text { Floor }(\mathrm{m})\end{array}$} & \multirow[b]{2}{*}{ Lithology } & \multirow[b]{2}{*}{ Age } & \multicolumn{3}{|c|}{$\begin{array}{c}\text { Bulk Sample } \\
\text { Major Constituent }\end{array}$} & \multicolumn{3}{|c|}{$\begin{array}{l}2-20 \mu \mathrm{m} \text { Fraction } \\
\text { Major Constituent }\end{array}$} & \multicolumn{3}{|c|}{$\begin{array}{l}<2 \mu \mathrm{m} \text { Fraction } \\
\text { Major Constituent }\end{array}$} \\
\hline & & & & 1 & 2 & 3 & 1 & 2 & 3 & 1 & 2 & 3 \\
\hline $\begin{array}{l}1-1,110 \\
2-2,37 \\
3-1,98\end{array}$ & $\begin{array}{r}1.1 \\
14.4 \\
41.5\end{array}$ & $\begin{array}{l}\text { Altered volcanic } \\
\text { ash and biogenous } \\
\text { volcanic silt }\end{array}$ & $\begin{array}{c}\text { Early } \\
\text { Eocene }\end{array}$ & $\begin{array}{l}\text { K-Fe. } \\
\text { Calc. } \\
\text { Calc. }\end{array}$ & $\begin{array}{l}\text { Mont. } \\
\text { Mont. } \\
\text { Pyri. }\end{array}$ & $\begin{array}{l}\text { Plag. } \\
\text { K-Fe. }\end{array}$ & $\begin{array}{l}\text { K-Fe. } \\
\text { Clin. } \\
\text { Pyri. }\end{array}$ & $\begin{array}{l}\text { Plag. } \\
\text { K-Fe. } \\
\text { K-Fe. }\end{array}$ & $\begin{array}{l}\text { Augi. } \\
\text { Plag. } \\
\text { Ilme. }\end{array}$ & $\begin{array}{l}\text { Mont. } \\
\text { Mont. } \\
\text { Mont. }\end{array}$ & $\begin{array}{l}\text { K-Fe. } \\
\text { Pyri. } \\
\text { Pyri. }\end{array}$ & \\
\hline
\end{tabular}

TABLE 7

Summary of X-Ray Mineralogy Samples, Sample Depths, Lithology, Age, and X-Ray Diffraction Results, Site 310

\begin{tabular}{|c|c|c|c|c|c|c|c|c|c|c|c|c|}
\hline \multirow[t]{2}{*}{$\begin{array}{c}\text { Sample } \\
\text { (Interval in } \mathrm{cm} \text { ) }\end{array}$} & \multirow{2}{*}{$\begin{array}{l}\text { Sample Depth } \\
\text { Below Sea } \\
\text { Floor }(\mathrm{m})\end{array}$} & \multirow[b]{2}{*}{ Lithology } & \multirow[b]{2}{*}{ Age } & \multicolumn{3}{|c|}{$\begin{array}{c}\text { Bulk Sample } \\
\text { Major Constituent }\end{array}$} & \multicolumn{3}{|c|}{$\begin{array}{l}2-20 \mu \mathrm{m} \text { Fraction } \\
\text { Major Constituent }\end{array}$} & \multicolumn{3}{|c|}{$\begin{array}{l}<2 \mu \mathrm{m} \text { Fraction } \\
\text { Major Constituent }\end{array}$} \\
\hline & & & & 1 & 2 & 3 & 1 & 2 & 3 & 1 & 2 & 3 \\
\hline \multicolumn{13}{|l|}{ Hole 310} \\
\hline $\begin{array}{l}1-1,5 \\
1-2,100 \\
3-2,99 \\
4-2,41 \\
5-6,32 \\
6-5,130 \\
8-5,100\end{array}$ & $\begin{array}{r}0.1 \\
2.5 \\
17.0 \\
25.9 \\
41.3 \\
50.3 \\
69.0\end{array}$ & $\begin{array}{c}\text { Unit } 1 \\
\text { Radiolarian- } \\
\text { bearing nanno } \\
\text { ooze }\end{array}$ & $\begin{array}{c}\text { Late } \\
\text { Miocene } \\
\text { to } \\
\text { Quaternary }\end{array}$ & $\begin{array}{l}\text { Calc. } \\
\text { Calc. } \\
\text { Calc. } \\
\text { Calc. } \\
\text { Calc. } \\
\text { Calc. } \\
\text { Calc. }\end{array}$ & $\begin{array}{l}\text { Quar. } \\
\text { Mica } \\
\text { Quar. } \\
\text { Mica } \\
\text { Quar. }\end{array}$ & $\begin{array}{l}\text { Quar. } \\
\text { Mica } \\
\text { Quar. } \\
\text { Mica }\end{array}$ & $\begin{array}{l}\text { Quar. } \\
\text { Quar. } \\
\text { Mica } \\
\text { Quar. } \\
\text { Mica } \\
\text { Mica } \\
\text { Mica }\end{array}$ & $\begin{array}{l}\text { Mica } \\
\text { Mica } \\
\text { Quar. } \\
\text { Mica } \\
\text { Quar. } \\
\text { Quar. } \\
\text { Quar. }\end{array}$ & $\begin{array}{l}\text { Plag. } \\
\text { Plag. } \\
\text { Plag. } \\
\text { Plag. } \\
\text { Plag. } \\
\text { Plag. } \\
\text { Plag. }\end{array}$ & $\begin{array}{l}\text { Mica } \\
\text { Mica } \\
\text { Mica } \\
\text { Mica } \\
\text { Mica } \\
\text { Mica } \\
\text { Paly. }\end{array}$ & $\begin{array}{l}\text { Quar. } \\
\text { Mont. } \\
\text { Quar. } \\
\text { Mont. } \\
\text { Quar. } \\
\text { Quar. } \\
\text { Quar. }\end{array}$ & $\begin{array}{l}\text { Plag. } \\
\text { Quar. } \\
\text { Mont. } \\
\text { Quar. } \\
\text { Mont. } \\
\text { Mont. } \\
\text { Mica }\end{array}$ \\
\hline $\begin{array}{l}9-6,5 \\
10-6,101\end{array}$ & $\begin{array}{l}79.1 \\
89.0\end{array}$ & Unit $2^{\mathrm{a}}$ & $\mathrm{a}$ & $\begin{array}{l}\text { Calc, } \\
\text { Calc. }\end{array}$ & $\begin{array}{l}\text { Quar. } \\
\text { Phil. }\end{array}$ & $\begin{array}{l}\text { Phil. } \\
\text { K-Fe. }\end{array}$ & $\begin{array}{l}\text { Mica } \\
\text { Phil. }\end{array}$ & $\begin{array}{l}\text { Quar. } \\
\text { K-Fe. }\end{array}$ & Phil. & $\begin{array}{l}\text { Phil. } \\
\text { Mont. }\end{array}$ & $\begin{array}{l}\text { Mica } \\
\text { Apat. }\end{array}$ & $\begin{array}{l}\text { Quar. } \\
\text { K-Fe. }\end{array}$ \\
\hline $\begin{array}{l}13-6,98 \\
\text { Hole } 310 A\end{array}$ & 117.5 & Unit $4^{b}$ & b & Calc. & Clin. & & Clin. & Mica & & Mont. & Paly. & Mica. \\
\hline $17-7,0$ & 334.0 & Unit $5^{c}$ & c & Bari. & Pyri. & Trid. & Bari. & Pyri. & Trid. & Mont. & Cris. & Paly. \\
\hline
\end{tabular}

a Zeolitic, nanno ooze; and nanno ooze; Oligocene and middle Miocene

${ }^{b}$ Nanno ooze; Maestrichtian to late Campanian.

${ }^{c}$ Chert and nanno ooze; Campanian 
TABLE 8

Summary of X-Ray Mineralogy Samples, Sample Depths, Lithology, Age, and X-Ray Diffraction Results, Site 311

\begin{tabular}{|c|c|c|c|c|c|c|c|c|c|c|c|c|}
\hline \multirow{2}{*}{$\begin{array}{c}\text { Sample } \\
\text { (Interval in } \mathrm{cm} \text { ) }\end{array}$} & \multirow{2}{*}{$\begin{array}{l}\text { Sample Depth } \\
\text { Below Sea } \\
\text { Floor (m) }\end{array}$} & \multirow[b]{2}{*}{ Lithology } & \multirow[b]{2}{*}{ Age } & \multicolumn{3}{|c|}{$\begin{array}{c}\text { Bulk Sample } \\
\text { Major Constituent }\end{array}$} & \multicolumn{3}{|c|}{$\begin{array}{l}2-20 \mu \mathrm{m} \text { Fraction } \\
\text { Major Constituent }\end{array}$} & \multicolumn{3}{|c|}{$\begin{array}{l}<2 \mu \mathrm{m} \text { Fraction } \\
\text { Major Constituent }\end{array}$} \\
\hline & & & & 1 & 2 & 3 & 1 & 2 & 3 & 1 & 2 & 3 \\
\hline $\begin{array}{l}1-6,142 \\
1-2,99 \\
2-3,106 \\
2-5,80 \\
\end{array}$ & $\begin{array}{r}8.9 \\
2.5 \\
13.1 \\
15.8 \\
\end{array}$ & $\begin{array}{c}\text { Unit } 1 \\
\text { Zeolitic pelagic } \\
\text { clay }\end{array}$ & $\begin{array}{c}\text { Late } \\
\text { Oligocene }\end{array}$ & $\begin{array}{l}\text { Augi. } \\
\text { Quar. } \\
\text { K-Fe. } \\
\text { Calc. }\end{array}$ & $\begin{array}{l}\text { Plag. } \\
\text { Mica. } \\
\text { Phil. } \\
\text { K-Fe. }\end{array}$ & $\begin{array}{l}\text { Phil. } \\
\text { Plag. } \\
\text { Quar. } \\
\text { Plag. }\end{array}$ & $\begin{array}{l}\text { Plag. } \\
\text { Mica } \\
\text { K-Fe. } \\
\text { K-Fe. }\end{array}$ & $\begin{array}{l}\text { Augi. } \\
\text { Quar. } \\
\text { Phil. } \\
\text { Magn. }\end{array}$ & $\begin{array}{l}\text { Phil. } \\
\text { Plag. } \\
\text { Mica } \\
\text { Plag. }\end{array}$ & $\begin{array}{l}\text { Mont. } \\
\text { Mica } \\
\text { Mont. } \\
\text { Mont. }\end{array}$ & $\begin{array}{l}\text { Augi. } \\
\text { Mont. } \\
\text { K-Fe. } \\
\text { Phil. }\end{array}$ & $\begin{array}{l}\text { Quar. } \\
\text { Quar. } \\
\text { K-Fe. }\end{array}$ \\
\hline $4-7,0$ & 24.0 & Unit $2^{\mathrm{a}}$ & $a$ & Mont. & Phil. & & Mont. & Phil. & & Mont. & Phil. & \\
\hline
\end{tabular}

${ }^{\mathrm{a}}$ Volcanic turbidites; age unknown.

TABLE 9

Summary of X-Ray Mineralogy Samples, Sample Depths, Lithology, Age, and X-Ray Diffraction Results, Site 313

\begin{tabular}{|c|c|c|c|c|c|c|c|c|c|c|c|c|}
\hline \multirow{2}{*}{$\begin{array}{c}\text { Sample } \\
\text { (Interval in } \mathrm{cm} \text { ) }\end{array}$} & \multirow{2}{*}{$\begin{array}{l}\text { Sample Depth } \\
\text { Below Sea } \\
\text { Floor }(\mathrm{m})\end{array}$} & \multirow[b]{2}{*}{ Lithology } & \multirow[b]{2}{*}{ Age } & \multicolumn{3}{|c|}{$\begin{array}{c}\text { Bulk Sample } \\
\text { Major Constituent }\end{array}$} & \multicolumn{3}{|c|}{$\begin{array}{l}2-20 \mu \mathrm{m} \text { Fraction } \\
\text { Major Constituent }\end{array}$} & \multicolumn{3}{|c|}{$\begin{array}{l}<2 \mu \mathrm{m} \text { Fraction } \\
\text { Major Constituent }\end{array}$} \\
\hline & & & & $T$ & 2 & 3 & 1 & 2 & 3 & 1 & 2 & 3 \\
\hline $\begin{array}{l}1-2,99 \\
1-5,49 \\
2-2,99 \\
3-2,101 \\
3-5,99 \\
4-5,99 \\
5-2,69 \\
7-5,89 \\
12-2,98 \\
13-2,99 \\
15-2,49 \\
19-5,100 \\
22-2,120\end{array}$ & $\begin{array}{r}2.5 \\
6.5 \\
38.0 \\
76.0 \\
80.5 \\
118.5 \\
151.7 \\
174.9 \\
207.5 \\
216.5 \\
234.5 \\
305.5 \\
399.7\end{array}$ & $\begin{array}{c}\text { Unit 1 } \\
\text { Foram-nanno } \\
\text { ooze, rad nanno } \\
\text { ooze, zeolitic } \\
\text { nanno ooze. and } \\
\text { chalks and cherts }\end{array}$ & $\begin{array}{l}\text { Quaternary } \\
\text { to } \\
\text { early } \\
\text { Maestrich- } \\
\text { tian }\end{array}$ & $\begin{array}{l}\text { Calc. } \\
\text { Calc. } \\
\text { Calc. } \\
\text { Calc. } \\
\text { Calc. } \\
\text { Calc. } \\
\text { Calc. } \\
\text { Calc. } \\
\text { Calc. } \\
\text { Calc. } \\
\text { Calc. } \\
\text { Calc. } \\
\text { Calc. }\end{array}$ & Quar. & Plag. & $\begin{array}{l}\text { Mica } \\
\text { Mica } \\
\text { Plag. } \\
\text { Clin. } \\
\text { Ins } \\
\text { Ins } \\
\text { Clin. } \\
\text { Clin. } \\
\text { Ins } \\
\text { K-Fe. } \\
\text { Bari. } \\
\text { Clin. } \\
\text { Clin. }\end{array}$ & $\begin{array}{l}\text { Quar. } \\
\text { Plag. } \\
\text { Phil. } \\
\text { Phil. } \\
\text { ficient re } \\
\text { ficient re } \\
\text { Plag. } \\
\text { Plag. } \\
\text { ficient re } \\
\text { Bari. } \\
\text { Clin. } \\
\text { Mont. } \\
\text { Cris. }\end{array}$ & $\begin{array}{l}\text { Plag. } \\
\text { Quar. } \\
\text { Quar. } \\
\text { Plag. } \\
\text { due } \\
\text { due } \\
\text { K-Fe. } \\
\text { Mont. } \\
\text { due } \\
\text { Quar. } \\
\text { Quar. } \\
\text { Plag. } \\
\text { K-Fe. }\end{array}$ & $\begin{array}{l}\text { Mica } \\
\text { Mica } \\
\text { Mont. } \\
\text { Mont. } \\
\text { Mont. } \\
\text { Mont. } \\
\text { Mont. } \\
\text { Mont. } \\
\text { Mont. } \\
\text { Mont. } \\
\text { Mont. } \\
\text { Mont. } \\
\text { Mont. }\end{array}$ & $\begin{array}{l}\text { Phil. } \\
\text { Mont. } \\
\text { Plag. } \\
\text { Clin. } \\
\text { Phil. } \\
\text { Clin. } \\
\text { Clin. } \\
\text { Kaol. } \\
\text { Plag. } \\
\text { K-Fe. } \\
\text { Paly. } \\
\text { Mica. } \\
\text { Paly. }\end{array}$ & $\begin{array}{l}\text { Quar. } \\
\text { Plag. } \\
\text { Phil. } \\
\text { Clin. } \\
\text { Mica } \\
\text { Plag. } \\
\text { Plag. } \\
\text { K-Fe. } \\
\text { Quar. } \\
\text { Quar. } \\
\text { Quar. } \\
\text { Cris. }\end{array}$ \\
\hline $\begin{array}{l}23-7,0 \\
24-3,83 \\
24-5,110 \\
28-4,54 \\
30-1,61 \\
31-2,66 \\
33-2,85 \\
35-4,84 \\
35-5,95 \\
41-5,147 \\
42-3,130 \\
42-3,145\end{array}$ & $\begin{array}{l}409.0 \\
413.3 \\
416.6 \\
452.5 \\
466.6 \\
477.7 \\
496.9 \\
518.8 \\
520.5 \\
576.5 \\
582.8 \\
583.0\end{array}$ & $\begin{array}{c}\text { Unit } 2 \\
\text { Foram nanno } \\
\text { limestone and } \\
\text { calcareous } \\
\text { volcanic sand- } \\
\text { stone, siltstone, } \\
\text { claystone and } \\
\text { breccia in turbidite } \\
\text { sequences }\end{array}$ & $\begin{array}{c}\text { early } \\
\text { Maestrich- } \\
\text { tian to } \\
\text { Companian }\end{array}$ & $\begin{array}{l}\text { Calc. } \\
\text { Calc. } \\
\text { Mont. } \\
\text { Calc. } \\
\text { Calc. } \\
\text { Arag. } \\
\text { Mont. } \\
\text { Mont. } \\
\text { Calc. } \\
\text { Calc. } \\
\text { Mont. } \\
\text { Calc. }\end{array}$ & $\begin{array}{l}\text { Mont. } \\
\text { Calc. } \\
\text { Mont. } \\
\text { K-Fe. } \\
\text { Calc. } \\
\text { Calc. } \\
\text { Calc. } \\
\\
\text { Calc. }\end{array}$ & $\begin{array}{l}\text { Augi. } \\
\text { Mont. } \\
\text { Phil. }\end{array}$ & $\begin{array}{l}\text { Augi. } \\
\text { K-Fe. } \\
\text { Phil. } \\
\text { Phil. } \\
\text { K-Fe. } \\
\text { Clin. } \\
\text { Mont. } \\
\text { Mont. } \\
\text { Phil. } \\
\text { K-Fe. } \\
\text { Mont. } \\
\text { Clin. }\end{array}$ & $\begin{array}{l}\text { Mont. } \\
\text { Hema. } \\
\text { Mont. } \\
\text { K-Fe. } \\
\text { Plag. } \\
\text { K-Fe. } \\
\text { Phil. } \\
\text { Phil. } \\
\text { K-Fe. } \\
\text { Plag. } \\
\text { K-Fe. } \\
\text { Bari. }\end{array}$ & $\begin{array}{l}\text { Plag. } \\
\text { Mica } \\
\text { Anal. } \\
\text { Mont. } \\
\\
\text { Plag. } \\
\text { K-Fe. } \\
\text { K-Fe. } \\
\text { Augi. } \\
\text { Mica. } \\
\text { Magn. } \\
\text { K-Fe. }\end{array}$ & $\begin{array}{l}\text { Mont. } \\
\text { Mont. } \\
\text { Mont. } \\
\text { Mont. } \\
\text { Mont. } \\
\text { Mont. } \\
\text { Mont. } \\
\text { Mont. } \\
\text { Mont. } \\
\text { Mont. } \\
\text { Mont. } \\
\text { Mont. }\end{array}$ & $\begin{array}{l}\text { Mica } \\
\text { Phil. } \\
\text { K-Fe. } \\
\text { Phil. }\end{array}$ & $\begin{array}{l}\text { Plag. } \\
\text { Bari }\end{array}$ \\
\hline
\end{tabular}

TABLE 10

Results of X-Ray Diffraction Analysis, Site 303

\begin{tabular}{|c|c|c|c|c|c|c|c|c|c|c|c|c|c|}
\hline Core & $\begin{array}{c}\text { Cored } \\
\text { Interval } \\
\text { Below Sea } \\
\text { Floor }(\mathrm{m})\end{array}$ & $\begin{array}{l}\text { Sample } \\
\text { Depth } \\
\text { Below Sea } \\
\text { Floor }(\mathrm{m})\end{array}$ & है & లే & 䒕 & 它 & 递 & $\begin{array}{l}\dot{0} \\
\ddot{g}\end{array}$ & 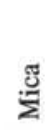 & ㅇํㄹ & 䒕 & 苂 & 脊 \\
\hline
\end{tabular}

Bulk Samples

$\begin{array}{lc}2 & 62.0-71.0 \\ 3 & 117.0-126.0\end{array}$

$5 \mathrm{~A} \quad 247.8-257.0$

65.2

124.4

250.5
94.5
87.6

77.1 $\begin{array}{cc}4.6 & 40.1 \\ - & 37.3\end{array}$

$1.7 \quad 13.5$

\section{$-\quad 25.5$}

$\begin{array}{rr}4.4 & 18.7 \\ 4.8 & 6.3\end{array}$

6.3

\section{-24.6
$-\quad 27.3$}

$\begin{array}{lr}3.3 & 27.3 \\ -\quad 10.5\end{array}$

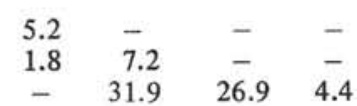

\section{2-20 $\mu \mathrm{m}$ Fraction}

$2 \quad 62.0-71.0$

$3 \quad 117.0-126.0$

65.20

88.3

124.4

$174.0-183.0$

176.3

5A $\quad 247.8-257.0$

250.5

\section{4}

$64.8-42.6$

$-\quad 23.3$
4.1

$\begin{array}{crr}- & 29.8 & 5.0 \\ 1.4 & 27.1 & 3.7 \\ 0.7 & 32.3 & 1.1\end{array}$

$\begin{array}{ccc}- & - & - \\ - & - & - \\ \overline{10.3} & 56.6 & 3.6\end{array}$

\section{$<2 \mu \mathrm{m}$ Fractions}

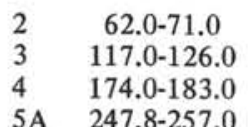

65.2

124.4

176.3

250.5

$\begin{array}{rrrr}80.5 & - & 25.1 & \\ 62.0 & - & 18.4 & 2.3 \\ 57.0 & - & 23.6 & 2.5 \\ 57.7 & 4.6 & 4.6 & 6.4\end{array}$

-
2.3
2.5
6.4

13.2

7.7

11.4
4.7

$\begin{array}{llll}4.1 & 25.7 & 5.3 & 26.5\end{array}$

$\begin{array}{llll}3.6 & 27.8 & 3.6 & 36.6\end{array}$

57.7 
TABLE 11

Results of X-Ray Diffraction Analysis, Site 304

\begin{tabular}{|c|c|c|c|c|c|c|c|c|c|c|c|}
\hline Core & $\begin{array}{c}\text { Cored } \\
\text { Interval } \\
\text { Below Sea } \\
\text { Floor (m) }\end{array}$ & $\begin{array}{l}\text { Sample } \\
\text { Depth } \\
\text { Below Sea } \\
\text { Floor }(\mathrm{m})\end{array}$ & 客 & हี & 它 & 迥 & 迥 & 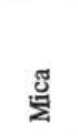 & है & 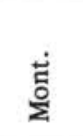 & घं \\
\hline \multicolumn{12}{|c|}{ Bulk Samples } \\
\hline $\begin{array}{l}1 \\
2\end{array}$ & $\begin{array}{l}105.5-115.0 \\
216.0-225.5\end{array}$ & $\begin{array}{l}110.1 \\
218.4\end{array}$ & $\begin{array}{l}94.8 \\
65.4\end{array}$ & $\begin{array}{l}46.5 \\
25.8\end{array}$ & - & $\begin{array}{l}18.2 \\
11.9\end{array}$ & $\begin{array}{l}4.4 \\
2.2\end{array}$ & $\begin{array}{l}23.0 \\
21.5\end{array}$ & $\begin{array}{l}4.5 \\
-\end{array}$ & $\begin{array}{r}3.3 \\
38.7\end{array}$ & - \\
\hline \multicolumn{12}{|c|}{$2-20 \mu \mathrm{m}$ Fractions } \\
\hline $\begin{array}{l}1 \\
2\end{array}$ & $\begin{array}{l}105.5-115.0 \\
216.0-225.5\end{array}$ & $\begin{array}{l}110.1 \\
218.4\end{array}$ & $\begin{array}{l}81.9 \\
48.1\end{array}$ & $\begin{array}{l}38.9 \\
37.9\end{array}$ & $\begin{array}{l}6.1 \\
-\end{array}$ & $\begin{array}{l}23.0 \\
25.7\end{array}$ & 2.7 & $\begin{array}{l}24.8 \\
32.7\end{array}$ & $\begin{array}{l}7.2 \\
-\end{array}$ & - & $\overline{1.1}$ \\
\hline \multicolumn{12}{|c|}{$<2 \mu \mathrm{m}$ Fractions } \\
\hline $\begin{array}{l}1 \\
2\end{array}$ & $\begin{array}{l}105.5-115.0 \\
216.0-225.5\end{array}$ & $\begin{array}{l}110.1 \\
218.4\end{array}$ & $\begin{array}{l}72.9 \\
50.1\end{array}$ & $\begin{array}{l}22.2 \\
12.6\end{array}$ & - & $\begin{array}{r}10.4 \\
4.3\end{array}$ & $\begin{array}{l}6.2 \\
4.2\end{array}$ & $\begin{array}{l}30.3 \\
14.8\end{array}$ & $\begin{array}{l}7.1 \\
-\end{array}$ & $\begin{array}{l}23.8 \\
64.0\end{array}$ & - \\
\hline
\end{tabular}

TABLE 12

Results of X-Ray Diffraction Analysis, Site 305

\begin{tabular}{|c|c|c|c|c|c|c|c|c|c|c|c|c|c|c|c|c|c|c|c|c|c|c|}
\hline Core & $\begin{array}{l}\text { Cored } \\
\text { Interval } \\
\text { Below Sea } \\
\text { Floor (m) }\end{array}$ & $\begin{array}{l}\text { Sample } \\
\text { Depth } \\
\text { Below Sea } \\
\text { Floor }(\mathrm{m})\end{array}$ & 安 & 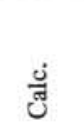 & 己ี & 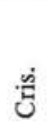 & 㟝 & $\frac{a d}{2 \pi}$ & $\begin{array}{l}\dot{0} \\
\ddot{g}\end{array}$ & $\stackrel{\Xi}{\Sigma}$ & 完 & 荒 & 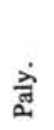 & 节 & छ & 咅 & 䓌 & $\stackrel{\mathrm{g}}{\mathrm{a}}$ & 㞓 & 竞 & 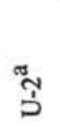 & $\stackrel{\rho}{s}$ \\
\hline \multicolumn{23}{|c|}{ Bulk Samples } \\
\hline 4 & $26.5-35.5$ & 29.9 & 31.7 & 98.3 & 1.0 & - & 0.8 & - & - & - & - & - & - & - & - & - & - & - & - & - & - & - \\
\hline \multirow[t]{2}{*}{5} & $35.5-45.0$ & 38.3 & 46.2 & 89.9 & 3.6 & - & - & 2.5 & - & 2.5 & - & - & - & - & - & - & - & - & 1.5 & - & - & - \\
\hline & & 40.8 & 53.5 & 75.7 & 5.7 & - & 3.3 & 3.9 & 1.6 & 7.6 & 1.2 & - & - & - & - & - & - & - & 1.0 & - & - & - \\
\hline \multirow[t]{3}{*}{6} & $45.0-54.5$ & 50.4 & 41.9 & 86.0 & 2.2 & - & 2.4 & 1.9 & - & 2.4 & - & - & - & - & - & 3.7 & - & - & 1.5 & - & - & - \\
\hline & & 51.2 & 34.5 & 91.1 & 1.2 & - & 1.4 & 1.4 & - & 1.2 & - & - & - & - & - & 1.9 & - & - & 1.8 & - & - & - \\
\hline & & 52.3 & 32.3 & 100.0 & - & - & - & - & - & - & - & - & - & - & - & - & - & - & - & - & - & - \\
\hline \multirow[t]{2}{*}{7} & $54.5-64.0$ & 57.0 & 18.5 & 100.0 & - & - & - & - & - & - & - & - & - & - & - & - & - & - & - & - & - & - \\
\hline & & 61.0 & 38.3 & 98.3 & 0.5 & - & - & - & - & - & - & - & - & - & - & 1.2 & - & - & - & - & - & - \\
\hline 8 & $64.0-73.0$ & 66.5 & 22.4 & 100.0 & - & - & - & - & - & - & - & - & - & - & - & - & - & - & - & - & - & - \\
\hline 9 & $73.0-82.0$ & 80.0 & 20.0 & 99.7 & 0.3 & - & - & - & - & - & - & - & - & - & - & - & - & - & - & - & - & _ \\
\hline 10 & $82.0-91.5$ & 89.0 & 15.5 & 100.0 & - & - & - & - & - & - & - & - & - & - & - & - & - & - & - & - & - & - \\
\hline 11 & $91.5-101.0$ & 93.7 & 17.7 & 100.0 & - & - & - & - & - & - & - & - & - & - & - & - & - & - & - & - & - & - \\
\hline 13 & $110.5-120.0$ & 113.0 & 20.8 & 100.0 & - & - & - & - & - & - & - & - & - & - & - & - & - & - & - & - & - & - \\
\hline 15 & $129.5-139.0$ & 136.5 & 14.8 & 100.0 & - & - & - & - & - & - & - & - & - & - & - & - & - & - & - & - & - & - \\
\hline \multirow[t]{2}{*}{16} & $139.0-148.5$ & 141.5 & 17.3 & 100.0 & - & - & - & - & - & - & - & - & - & - & - & - & - & - & - & - & - & - \\
\hline & & 145.0 & 22.3 & 100.0 & - & - & - & - & - & - & - & - & - & - & - & - & - & - & - & - & - & - \\
\hline 17 & $148.5-158.0$ & 155.5 & 35.5 & 100.0 & - & - & - & - & - & - & - & - & - & - & - & - & - & - & - & - & - & - \\
\hline 20 & $176.5-186.0$ & 183.5 & 19.2 & 100.0 & - & - & - & - & - & - & - & - & - & - & - & - & - & - & - & - & - & - \\
\hline 23 & $205.0-214.0$ & 212.0 & 18.9 & 100.0 & - & - & - & - & - & - & - & - & - & - & - & - & - & - & - & - & - & - \\
\hline 25 & $223.5-233.0$ & 230.5 & 28.7 & 100.0 & - & - & - & - & - & - & - & - & - & - & - & - & - & - & - & - & - & - \\
\hline
\end{tabular}




\begin{tabular}{|c|c|c|c|c|c|c|c|c|c|c|c|c|c|c|c|c|c|c|c|c|c|c|}
\hline Core & $\begin{array}{c}\text { Cored } \\
\text { Interval } \\
\text { Below Sea } \\
\text { Floor (m) }\end{array}$ & $\begin{array}{c}\text { Sample } \\
\text { Depth } \\
\text { Below Sea } \\
\text { Floor (m) }\end{array}$ & 总 & లू & $\frac{\mathfrak{g}}{\partial}$ & 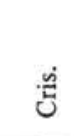 & 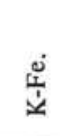 & $\frac{\text { of }}{2}$ & 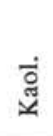 & $\frac{g}{\Sigma}$ & 宅 & $\frac{\tilde{\sigma}}{2}$ & $\frac{2}{a}$ & $\underset{H}{\vec{E}}$ & 实 & 言 & 岕 & $\overrightarrow{\mathrm{z}}$ & 㟧 & 言 & $\stackrel{\pi}{\text { Tึ }}$ & ? \\
\hline 59 & $550.5-560.0$ & 551.8 & 34.9 & 38.4 & 60.5 & - & - & - & - & 1.1 & - & - & - & - & - & - & - & - & - & - & - & - \\
\hline 60 & $560.0-569.5$ & $\begin{array}{l}551.9 \\
561.0\end{array}$ & $\begin{array}{l}32.8 \\
334\end{array}$ & $\begin{array}{l}93.3 \\
46.8\end{array}$ & 53.1 & - & - & - & - & - & - & - & - & - & - & - & - & - & - & - & - & - \\
\hline 65 & $607.0-616.5$ & 608.0 & $\begin{array}{l}32.4 \\
52.0\end{array}$ & $\begin{array}{r}4.8 \\
2.5\end{array}$ & $\begin{array}{l}53.2 \\
64.3\end{array}$ & 26.5 & $\overline{-}$ & $\overline{-}$ & $=$ & $\overline{-}$ & $\overline{-}$ & $\overline{-}$ & 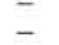 & $\overline{2.5}$ & $\overline{-}$ & - & 2.7 & $\overline{-}$ & $\overline{1.6}$ & $\begin{array}{l}- \\
-\end{array}$ & $\overline{-}$ & $\overline{-}$ \\
\hline 66 & $6165-6060$ & 608.3 & 47.8 & -0 & 16.3 & 62.8 & - & - & - & 2.1 & - & 5.7 & $-\overline{25}$ & 10.5 & 1.7 & - & 0.9 & - & - & - & - & - \\
\hline
\end{tabular}

2-20 $\mu \mathrm{m}$ Fractions

\begin{tabular}{|c|c|c|c|c|c|c|c|c|c|c|c|c|c|c|c|c|c|c|c|c|c|c|}
\hline 4 & $26.5-35.5$ & 29.9 & 88.7 & - & 34.7 & - & 7.6 & 19.2 & - & 33.6 & 4.9 & - & - & - & - & - & - & - & - & - & - & - \\
\hline 5 & $35.5-45.0$ & 38.3 & 46.5 & - & 36.7 & - & 5.9 & 18.0 & 2.0 & 33.5 & 3.9 & - & - & - & - & - & - & - & - & - & - & - \\
\hline & & 40.8 & 41.3 & - & 33.0 & - & 6.1 & 16.6 & 3.0 & 36.0 & 3.3 & - & - & - & - & - & - & - & 1.1 & 1.0 & - & - \\
\hline \multirow[t]{3}{*}{6} & $45.0-54.5$ & 50.4 & 32.8 & - & 20.8 & - & 8.3 & 13.4 & - & 19.0 & 2.9 & - & - & - & 1.5 & 28.5 & - & - & 5.6 & - & - & - \\
\hline & & 51.2 & 32.0 & - & 17.9 & - & 9.4 & 12.3 & - & 24.1 & 3.1 & - & - & - & - & 22.3 & - & - & 10.9 & - & - & - \\
\hline & & 52.3 & 77.4 & - & 19.0 & - & 8.7 & 13.4 & - & 18.3 & 2.3 & - & - & - & 1.1 & 33.1 & - & - & 4.1 & - & - & - \\
\hline \multirow[t]{2}{*}{7} & $54.5-64.0$ & 57.0 & 88.1 & - & 26.2 & - & 19.5 & 12.1 & - & 6.8 & 1.2 & - & - & - & - & 31.7 & - & - & 2.6 & - & - & - \\
\hline & & 61.0 & 68.5 & - & 15.8 & - & 6.7 & 8.8 & - & 4.2 & 1.3 & - & - & - & - & 63.2 & - & - & - & - & - & - \\
\hline 9 & $73.0-82.0$ & 80.0 & 89.0 & - & 31.8 & - & 24.7 & 11.4 & - & 8.7 & 2.3 & - & - & - & - & 19.5 & - & - & 1.6 & - & - & Trace \\
\hline 10 & $82.0-91.5$ & 89.0 & 82.5 & - & 28.8 & - & 22.7 & 15.7 & - & 13.4 & 2.4 & - & 4.8 & - & - & 8.3 & - & - & 4.0 & - & - & Trace \\
\hline 11 & $91.5-101.0$ & 93.7 & 94.3 & - & 9.5 & - & 68.8 & 17.7 & 1.3 & 1.0 & - & - & - & - & - & - & - & - & 1.7 & - & - & Trace \\
\hline 13 & $110.5-120.0$ & 113.0 & 81.2 & - & 29.3 & - & 60.9 & - & - & 5.9 & 0.9 & - & - & - & - & - & - & - & 3.1 & - & - & Pres \\
\hline 15 & $129.5-139.0$ & 36.5 & 92.6 & - & 33.4 & - & 54.7 & - & - & 7.5 & - & - & - & - & - & - & - & - & 4.4 & - & - & Trace \\
\hline 59 & $550.5-560.0$ & 551.9 & 42.2 & - & 46.7 & - & 7.1 & 3.5 & - & 21.6 & 1.9 & - & - & - & - & - & 0.6 & - & 18.5 & - & - & - \\
\hline \multirow[t]{2}{*}{65} & $607.0-616.5$ & 608.0 & 29.5 & - & 64.2 & 25.3 & - & - & - & - & - & - & - & 2.0 & - & - & 8.5 & - & - & - & Pres & - \\
\hline & & 608.3 & 25.8 & - & 23.5 & 50.9 & 5.6 & - & 2.6 & 1.5 & - & - & - & 7.6 & 6.5 & - & 1.8 & - & - & - & - & - \\
\hline 66 & $616.5-626.0$ & 617.7 & 29.5 & - & 88.7 & - & 1.8 & - & - & 6.5 & - & - & - & - & - & - & - & - & 3.0 & - & - & - \\
\hline \multicolumn{23}{|c|}{$<2 \mu \mathrm{m}$ Fractions } \\
\hline 4 & $26.5-35.5$ & 29.9 & 79.6 & - & 21.0 & - & 2.6 & 7.1 & 7.1 & 36.1 & 7.3 & 18.8 & - & - & - & - & - & - & - & - & - & - \\
\hline 5 & $35.5-45.0$ & 40.8 & 75.4 & - & 27.1 & - & 4.7 & 10.7 & 2.0 & 36.4 & 5.2 & 12.2 & - & - & - & - & - & - & 1.6 & - & - & - \\
\hline \multirow[t]{3}{*}{6} & $45.0-54.5$ & 50.4 & 73.7 & - & 23.5 & - & 5.9 & 9.0 & 3.5 & 32.9 & 4.2 & 13.0 & 7.0 & - & - & - & - & - & 0.9 & - & - & - \\
\hline & & 51.2 & 65.9 & - & 10.0 & - & 4.2 & 8.2 & 9.3 & 28.0 & 6.0 & 24.9 & 7.1 & - & - & - & - & - & 2.2 & - & - & - \\
\hline & & 52.3 & 89.4 & - & 12.8 & - & 3.6 & 6.9 & 4.1 & 7.3 & 7.5 & 3.4 & 45.8 & - & - & 8.6 & - & - & - & - & - & - \\
\hline \multirow[t]{3}{*}{7} & $54.5-64.0$ & 57.0 & 88.9 & - & 19.7 & - & 11.3 & 8.6 & 2.7 & 31.6 & 5.3 & 1.6 & 13.5 & - & - & 5.7 & - & - & - & - & - & - \\
\hline & & 61.0 & 83.3 & - & 17.7 & - & 10.7 & 3.7 & 3.1 & 34.9 & 6.5 & 5.5 & 7.3 & - & - & 10.6 & - & - & - & - & - & - \\
\hline & $64.0-73.0$ & 66.5 & 92.9 & - & 13.4 & - & 6.5 & 5.0 & 3.5 & 43.0 & 8.7 & 6.6 & 13.2 & - & - & - & - & - & - & - & - & - \\
\hline 9 & $73.0-82.0$ & 80.0 & 81.0 & - & 14.0 & - & 6.2 & - & 3.1 & 34.8 & 7.5 & 6.2 & 23.7 & - & - & 4.5 & - & - & - & - & - & - \\
\hline 10 & $82.0-91.5$ & 89.0 & 86.7 & - & 18.4 & - & 12.5 & 4.0 & 3.9 & 36.7 & 7.5 & 5.1 & 10.7 & - & - & - & - & 1.2 & - & - & - & - \\
\hline 11 & $91.5-101.0$ & 93.7 & 83.5 & - & 14.5 & - & 8.2 & 2.3 & 4.4 & 32.7 & 7.2 & 10.3 & 20.4 & - & - & - & - & - & - & - & - & - \\
\hline 13 & $110.5-120.0$ & 113.0 & 79.6 & - & 0.4 & - & 0.4 & 0.2 & - & - & 0.2 & 98.7 & - & - & - & - & - & - & - & - & - & Trace \\
\hline 15 & $129.5-139.0$ & 136.5 & 80.6 & - & 13.7 & - & 12.0 & - & - & 16.1 & 4.3 & 37.2 & 16.6 & - & - & - & - & - & - & - & - & - \\
\hline \multirow[t]{2}{*}{16} & $139.0-148.5$ & 141.5 & 83.7 & - & 11.5 & - & 9.7 & - & - & 16.9 & 3.9 & 53.0 & 5.0 & - & - & - & - & - & - & - & - & - \\
\hline & & 145.0 & 78.2 & - & 8.3 & - & 5.8 & 2.9 & - & 44.2 & 3.6 & 27.2 & 6.2 & - & - & - & - & - & 1.7 & - & - & - \\
\hline 17 & $148.5-158.0$ & 155.5 & 81.5 & - & 9.8 & - & 6.6 & 1.5 & 2.9 & 13.9 & 3.1 & 46.6 & 15.6 & - & - & - & - & - & - & - & - & - \\
\hline 20 & $176.5-186.0$ & 183.5 & 81.7 & - & 9.6 & - & 4.7 & - & 3.0 & 17.8 & 3.2 & 48.0 & 13.7 & - & - & - & - & - & - & - & - & - \\
\hline 23 & $205.0-214.0$ & 212.0 & 75.6 & - & 10.0 & - & 9.3 & - & 2.8 & 8.8 & 3.3 & 49.4 & 15.3 & - & - & - & - & - & 1.1 & - & - & - \\
\hline 59 & $550.5-560.0$ & 551.9 & 45.6 & - & 80.2 & - & 1.0 & - & - & 2.8 & - & 10.1 & 4.8 & - & - & - & - & - & 1.1 & - & - & - \\
\hline \multirow[t]{2}{*}{65} & $607.0-616.5$ & 608.0 & 34.5 & - & 54.8 & 42.9 & - & - & - & - & - & - & - & 1.1 & - & - & 1.2 & - & - & - & - & - \\
\hline & & 608.3 & 33.2 & - & 7.5 & 67.1 & 0.9 & 1.1 & - & 3.5 & - & 3.8 & 3.5 & 10.7 & 1.8 & - & - & - & - & - & - & - \\
\hline 66 & $616.5-626.0$ & 617.7 & 59.7 & - & 68.2 & - & 1.6 & - & - & 10.0 & 0.7 & 2.6 & 15.3 & - & - & - & - & - & 1.6 & - & - & - \\
\hline
\end{tabular}

${ }^{\mathrm{a}} \mathrm{U}-2$ identifiable peaks located at $1.755,2.683$, and $2.309 \AA$.

bU-3 identifiable peaks located at $1.933,1.972$, and $2.528 \AA$. 
TABLE 13

Results of X-Ray Diffraction Analysis, Site 306

\begin{tabular}{|c|c|c|c|c|c|c|c|c|c|c|c|c|c|c|c|c|}
\hline Core & $\begin{array}{c}\text { Cored } \\
\text { Interval } \\
\text { Below Sea } \\
\text { Floor (m) }\end{array}$ & $\begin{array}{l}\text { Sample } \\
\text { Depth } \\
\text { Below Sea } \\
\text { Floor (m) }\end{array}$ & 岕 & శึં & है & 总 & 兑 & $\frac{\approx}{2}$ & $\frac{\text { లే }}{\Sigma}$ & 을 & $\stackrel{\overrightarrow{0}}{\Sigma}$ & 总 & ह & $\overrightarrow{\dot{x}}$ & 吂 & 菅 \\
\hline \multicolumn{17}{|c|}{ Bulk Samples } \\
\hline 1 & $0.0-9.5$ & 2.8 & 53.4 & 84.9 & 8.6 & - & - & 1.7 & 4.8 & - & - & - & - & - & - & - \\
\hline 8 & $113.0-122.5$ & 114.0 & 33.6 & 46.8 & 11.5 & 30.3 & - & - & - & - & - & 11.5 & - & - & - & - \\
\hline \multirow[t]{2}{*}{21} & $281.0-290.5$ & 281.7 & 31.0 & 92.6 & 5.9 & - & - & - & - & - & - & - & - & - & 1.5 & - \\
\hline & & 282.4 & 33.1 & 90.5 & 7.3 & - & - & - & 1.3 & - & - & - & - & - & 0.9 & - \\
\hline 29 & $355.5-365.0$ & 364.5 & 31.4 & 85.7 & 14.3 & - & - & - & - & - & - & - & - & - & - & - \\
\hline 36 & $411.5-421.0$ & 420.5 & 30.1 & 97.8 & - & - & - & - & - & - & - & - & 0.5 & - & 1.8 & - \\
\hline 40 & $449.5-459.0$ & 450.9 & 43.5 & 96.6 & 0.5 & - & - & - & - & - & - & - & - & - & 2.9 & - \\
\hline \multicolumn{17}{|c|}{ 2-20 $\mu \mathrm{m}$ Fractions } \\
\hline 1 & $0.0-9.5$ & 2.8 & 42.9 & - & 33.0 & - & 5.3 & 24.4 & 30.6 & 5.7 & - & - & - & - & - & 1.0 \\
\hline 8 & $113.0-122.5$ & 114.0 & 26.9 & - & 25.2 & 54.3 & - & - & - & - & - & 20.5 & - & - & - & - \\
\hline \multirow[t]{2}{*}{21} & $281.0-290.5$ & 281.7 & 60.3 & - & 45.8 & - & 19.6 & 4.3 & 22.9 & 1.4 & - & - & 3.5 & - & 2.6 & - \\
\hline & & 282.4 & 38.5 & - & 52.3 & - & 11.4 & 4.3 & 22.8 & 1.5 & - & - & 3.6 & - & 4.0 & - \\
\hline 29 & $355.5-365.0$ & 364.5 & 28.4 & - & 86.1 & - & 2.0 & - & 5.0 & - & - & - & 2.3 & - & 4.6 & - \\
\hline 36 & $411.5-421.0$ & 420.5 & 33.4 & - & 13.2 & - & 23.6 & 2.4 & 11.3 & 1.9 & - & - & 14.5 & - & 33.2 & - \\
\hline 40 & $449.5-459.0$ & 450.9 & 35.4 & - & 15.0 & - & 12.4 & - & 18.2 & - & - & - & 13.2 & 6.8 & 34.4 & - \\
\hline \multicolumn{17}{|c|}{$<2 \mu \mathrm{m}$ Fractions } \\
\hline 1 & $0.0-9.5$ & 2.8 & 68.3 & - & 19.0 & - & - & 8.1 & 50.8 & 10.1 & 12.0 & - & - & - & - & - \\
\hline 8 & $113.0-122.5$ & 114.0 & 17.1 & - & 3.2 & 76.3 & - & - & 1.5 & - & - & 19.1 & - & - & - & - \\
\hline \multirow[t]{2}{*}{21} & $281.0-290.5$ & 281.7 & 47.4 & - & 88.4 & - & 1.9 & - & 7.0 & - & 2.7 & - & - & - & - & - \\
\hline & & 282.4 & 54.6 & - & 80.1 & - & 4.3 & - & 7.5 & - & 2.4 & - & - & 5.6 & - & - \\
\hline 29 & $355.5-365.0$ & 364.5 & 48.8 & - & 83.0 & - & 2.6 & - & 5.0 & - & - & - & 1.0 & 7.7 & 0.8 & - \\
\hline 36 & $411.5-421.0$ & 420.5 & 68.6 & - & 4.3 & - & 2.4 & - & 4.1 & - & 13.8 & - & 1.3 & 72.3 & 1.8 & - \\
\hline 40 & $449.5-459.0$ & 450.9 & 70.4 & - & 9.0 & - & - & - & - & - & - & - & 2.4 & 85.5 & 3.0 & - \\
\hline
\end{tabular}

TABLE 14

Results of X-Ray Diffraction Analysis, Site 307

\begin{tabular}{|c|c|c|c|c|c|c|c|c|c|c|c|c|c|c|c|c|c|c|c|c|}
\hline Core & $\begin{array}{c}\text { Cored } \\
\text { Interval } \\
\text { Below Sea } \\
\text { Floor }(\mathrm{m})\end{array}$ & $\begin{array}{l}\text { Sample } \\
\text { Depth } \\
\text { Below Sea } \\
\text { Floor (m) }\end{array}$ & $\stackrel{\text { Ĕ }}{<}$ & సुં & 응 & $\ddot{\tilde{z}}$ & $\frac{x}{2}$ & $\frac{\dot{v}}{\square}$ & $\frac{a b}{2}$ & $\begin{array}{l}\overrightarrow{0} \\
\ddot{g}\end{array}$ & $\frac{\tilde{g}}{\Sigma}$ & $\frac{\dot{0}}{\tilde{b}}$ & $\dot{\vec{\Xi}}$ & E & $\begin{array}{l}\text { Ė } \\
\text { Ė }\end{array}$ & 点 & $\frac{\vec{x}}{\Sigma}$ & 突 & 岇 & ङु \\
\hline
\end{tabular}

Bulk Samples

$\begin{array}{rc}1 & 0.0-9.0 \\ 5 & 103.0-112.5 \\ 9 & 232.5 \cdot 241.5 \\ 11 & 288.5-297.5\end{array}$

$\begin{array}{ll}11 & 288.5-297.5 \\ 12 & 297.5-307.0\end{array}$

2-20 $\mu \mathrm{m}$ Fractions

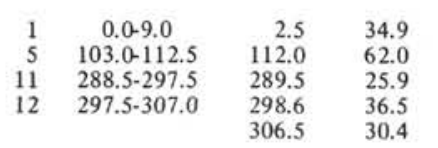

$\begin{array}{rrr}2.5 & 71.9 & \\ 112.0 & 78.2 & \\ 233.6 & 49.6 & 3.1 \\ 289.5 & 51.1 & \\ 298.6 & 67.0 & \\ 306.5 & 58.3 & \end{array}$

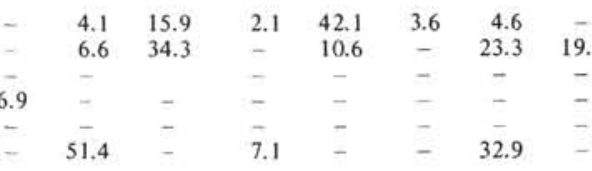

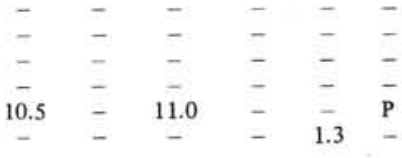

$<2 \mu \mathrm{m}$ Fractions

\begin{tabular}{|c|c|c|c|c|c|c|c|c|c|c|c|c|c|c|c|c|c|c|c|c|}
\hline 1 & $0.0-9.0$ & 2.5 & 66.2 & - & - & 16.6 & - & 4.9 & 8.8 & 2.9 & 39.0 & 6.7 & 21.1 & - & - & - & - & - & - & - \\
\hline 5 & $103.0-112.5$ & 112.0 & 62.6 & - & - & 4.1 & - & 6.0 & 13.2 & - & - & - & 73.5 & 1.1 & - & - & - & - & 2.0 & - \\
\hline 11 & $288.5-297.5$ & 289.5 & 51.7 & - & - & 59.2 & - & - & - & 19.9 & 11.4 & - & - & - & 9.5 & - & - & - & - & - \\
\hline 12 & $297.5-307.0$ & 298.6 & 70.7 & - & - & 37.9 & - & - & - & - & - & - & - & - & 28.9 & - & 33.2 & - & - & $\mathbf{P}$ \\
\hline & & 306.5 & 51.6 & - & - & 2.7 & - & 13.1 & - & 12.5 & - & - & 68.7 & - & - & 0.7 & - & - & 2.2 & - \\
\hline
\end{tabular}


TABLE 15

Results of X-Ray Diffraction Analysis, Site $\mathbf{3 0 8}$

\begin{tabular}{|c|c|c|c|c|c|c|c|c|c|c|c|c|c|c|c|c|c|}
\hline Core & $\begin{array}{c}\text { Cored } \\
\text { Interval } \\
\text { Below Sea } \\
\text { Floor }(\mathrm{m})\end{array}$ & $\begin{array}{l}\text { Sample } \\
\text { Depth } \\
\text { Below Sea } \\
\text { Floor (m) }\end{array}$ & 宫 & ฮું & 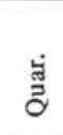 & $\frac{5}{4}$ & $\frac{\text { m }}{2}$ & 횸 & $\overrightarrow{\tilde{E}}$ & 坖 & है & 峁 & ह & 豆 & 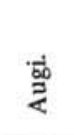 & $\stackrel{\pi}{5}$ & हีं \\
\hline \multicolumn{18}{|c|}{ Bulk Samples } \\
\hline $\begin{array}{l}1 \\
2 \\
3\end{array}$ & $\begin{array}{c}0.0-3.0 \\
12.5-21.5 \\
40.5-49.5\end{array}$ & $\begin{array}{r}1.1 \\
14.4 \\
41.5\end{array}$ & $\begin{array}{l}78.2 \\
64.2 \\
61.5\end{array}$ & $\begin{array}{r}6.9 \\
56.4 \\
85.9\end{array}$ & $\begin{array}{l}3.9 \\
- \\
-\end{array}$ & $\begin{array}{c}37.0 \\
10.3 \\
-\end{array}$ & $\begin{array}{c}16.7 \\
6.6 \\
-\end{array}$ & $\begin{array}{l}\overline{-} \\
\overline{-}\end{array}$ & $\begin{array}{r}23.8 \\
11.9 \\
3.4\end{array}$ & $\begin{array}{l}5.4 \\
6.0 \\
-\end{array}$ & $\begin{array}{l}- \\
-\end{array}$ & $\begin{array}{l}1.0 \\
2.9 \\
7.1\end{array}$ & $\begin{array}{l}- \\
\overline{0.6}\end{array}$ & $\begin{array}{l}- \\
5.9 \\
2.9\end{array}$ & $\begin{array}{l}5.4 \\
- \\
-\end{array}$ & $\begin{array}{l}- \\
\bar{T}\end{array}$ & $\overline{-}$ \\
\hline \multicolumn{18}{|c|}{$2-20 \mu \mathrm{m}$ Fractions } \\
\hline $\begin{array}{l}1 \\
2 \\
3\end{array}$ & $\begin{array}{c}0.0-3.0 \\
12.5-21.5 \\
40-5-49.5\end{array}$ & $\begin{array}{r}1.1 \\
14.4 \\
41.5\end{array}$ & $\begin{array}{l}43.2 \\
41.7 \\
44.3\end{array}$ & $\begin{array}{l}- \\
-\end{array}$ & $\begin{array}{l}5.7 \\
2.9 \\
3.6\end{array}$ & $\begin{array}{l}37.0 \\
25.0 \\
21.9\end{array}$ & $\begin{array}{r}18.8 \\
11.5 \\
5.7\end{array}$ & $\begin{array}{l}1.4 \\
- \\
-\end{array}$ & $\begin{array}{l}6.1 \\
9.8 \\
-\end{array}$ & $\begin{array}{c}11.5 \\
33.8 \\
-\end{array}$ & $\frac{-}{6.2}$ & $\begin{array}{r}0.7 \\
10.7 \\
42.0\end{array}$ & $\frac{-}{5.7}$ & $\begin{array}{r}- \\
6.4 \\
14.8\end{array}$ & $\begin{array}{c}13.5 \\
- \\
-\end{array}$ & $\begin{array}{l}- \\
-\end{array}$ & $\begin{array}{l}5.3 \\
- \\
-\end{array}$ \\
\hline \multicolumn{18}{|c|}{$<2 \mu \mathrm{m}$ Fractions } \\
\hline $\begin{array}{l}1 \\
2 \\
3\end{array}$ & $\begin{array}{c}0.0-3.0 \\
12.5-21.5 \\
40.5-49.5\end{array}$ & $\begin{array}{r}1.1 \\
14.4 \\
41.5\end{array}$ & $\begin{array}{l}60.2 \\
68.0 \\
70.9\end{array}$ & $\begin{array}{l}- \\
-\end{array}$ & $\begin{array}{l}1.8 \\
- \\
0.9\end{array}$ & $\begin{array}{l}9.9 \\
3.8 \\
-\end{array}$ & $\begin{array}{l}2.3 \\
- \\
-\end{array}$ & $\begin{array}{l}- \\
\overline{7.0}\end{array}$ & $\begin{array}{l}76.3 \\
83.0 \\
74.3\end{array}$ & $\begin{array}{l}4.5 \\
1.6 \\
-\end{array}$ & $\begin{array}{l}- \\
-\end{array}$ & $\begin{array}{r}0.7 \\
11.5 \\
15.5\end{array}$ & $\begin{array}{l}- \\
-\end{array}$ & $\begin{array}{l}- \\
\overline{2.3}\end{array}$ & $\begin{array}{l}4.5 \\
- \\
-\end{array}$ & $\begin{array}{l}- \\
-\end{array}$ & - \\
\hline
\end{tabular}

${ }^{\mathrm{a}} \mathrm{U}-1$ identifiable peak located at 3.07A.

TABLE 16

Results of X-Ray Diffraction Analysis, Site 310

\begin{tabular}{|c|c|c|c|c|c|c|c|c|c|c|c|c|c|c|c|c|c|c|c|c|c|c|c|}
\hline Core & $\begin{array}{c}\text { Cored } \\
\text { Interval } \\
\text { Below Sea } \\
\text { Floor (m) }\end{array}$ & $\begin{array}{c}\text { Sample } \\
\text { Depth } \\
\text { Below Sea } \\
\text { Floor }(\mathrm{m})\end{array}$ & 总 & हैं & 莣 & 胥 & $\stackrel{\breve{\leftrightarrows}}{\breve{\Delta}}$ & 己. & $\frac{\varpi}{2}$ & 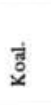 & $\frac{\pi}{2}$ & $\frac{\delta}{E}$ & $\frac{\vec{\partial}}{\frac{1}{z}}$ & हैं & $\vec{E}$ & है & 吾 & 怠 & 送 & 豞 & 言 & 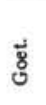 & 迎 \\
\hline \multicolumn{24}{|c|}{ Bulk Samples } \\
\hline 1 & $0.0-5.0$ & $\begin{array}{l}0.1 \\
2.5\end{array}$ & $\begin{array}{l}55.6 \\
60.4\end{array}$ & $\begin{array}{l}83.6 \\
62.8\end{array}$ & $=$ & $\begin{array}{r}6.3 \\
13.0\end{array}$ & $z$ & $=$ & $\begin{array}{l}3.4 \\
6.1\end{array}$ & $\bar{z}$ & $\begin{array}{r}4.9 \\
16.2\end{array}$ & $\begin{array}{l}1.8 \\
2.0\end{array}$ & $\overline{-}$ & $\overline{-}$ & $\overline{-}$ & $\overline{-}$ & $\overline{-}$ & $\overline{-}$ & $\bar{z}$ & $\overline{-}$ & $\bar{z}$ & $\bar{z}$ & $\bar{z}$ \\
\hline 3 & $14.5-24.0$ & 17.0 & 32.7 & 97.0 & - & 1.7 & - & - & - & - & 1.3 & - & - & - & - & - & - & - & - & - & - & - & - \\
\hline 4 & $24.0-33.5$ & 25.9 & 45.2 & 85.1 & - & 6.3 & 1.1 & - & 2.3 & $=$ & 5.2 & - & - & - & - & - & - & - & - & $=$ & - & - & - \\
\hline 5 & $33.5-43.0$ & 41.3 & 32.5 & 96.3 & - & 1.3 & - & - & & - & 1.7 & - & - & - & - & - & - & - & - & 0.7 & - & - & - \\
\hline 6 & $43.0-52.5$ & 50.3 & $\$ 2.3$ & 76.5 & - & 7.5 & & - & 3.4 & 1.2 & 8.3 & 1.1 & - & - & - & - & - & - & - & 0 & - & - & - \\
\hline 8 & $62.0-71.5$ & 69.0 & 49.8 & 80.3 & - & 7.8 & - & - & 2.7 & - & 5.6 & 1.3 & - & - & - & - & - & - & - & 3 & - & - & - \\
\hline 9 & $71.5-80.5$ & 79.1 & 52.0 & 59.3 & - & 11.9 & - & - & 5.2 & - & 9.1 & 0.9 & - & - & - & - & 9.6 & - & - & 3.9 & - & - & - \\
\hline 10 & $80.5-90.0$ & 89.0 & 58.4 & 41.0 & - & 5.7 & 14.7 & - & - & - & 3.3 & - & 4.5 & - & - & -0 & 30.8 & - & - & - & - & - & - \\
\hline 13 & $109.0-118.5$ & 117.5 & 31.3 & 88.9 & - & 4.3 & - & - & - & - & 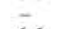 & - & - & 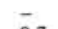 & - & 6.8 & - & - & - & - & - & - & - \\
\hline $17 \mathrm{~A}$ & $325.0-334.0$ & 334.0 & 55.9 & - & - & 9.9 & - & 13.1 & 2.8 & - & 6.6 & - & - & 8.7 & 13.3 & 2.5 & - & 14.2 & - & 28.8 & - & - & - \\
\hline \multicolumn{24}{|c|}{$2.20 \mu \mathrm{m}$ Fractions } \\
\hline 1 & $0.0-5.0$ & 0.1 & 52.5 & - & 5. & 39.3 & & & 2 & - & 29.5 & 5.7 & - & - & - & - & - & - & - & - & 0.9 & - & - \\
\hline & & 2 & 47.0 & - & 4. & 36.0 & 3.3 & & 19.2 & - & & & - & 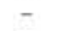 & - & - & - & - & 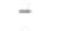 & - & 0.9 & - & - \\
\hline 3 & 14.5-24.0 & 17.0 & 51.9 & - & 8. & 31.3 & 3.5 & - & 14.4 & - & 35 & 6.3 & - & - & - & - & - & - & - & - & - & - & - \\
\hline 4 & $24.0-3$ & 25 & & - & 5 . & 34. & 4.7 & - & 17 & - & & & - & - & - & - & - & - & - & -3 & 1.1 & - & - \\
\hline 5 & $33.5-4$ & 41 & 54.3 & - & 8. & 29.9 & 2.8 & - & 14.6 & - & 36.4 & 6. & - & - & - & - & - & - & $\bar{z}$ & 1.3 & - & - & $\overline{-}$ \\
\hline 6 & $43.0-5$ & 50.3 & 55.8 & - & 4.0 & 32. & . & - & 15 & - & & & - & - & - & - & - & - & $\overline{-}$ & 8 & $\overline{3}$ & - & - \\
\hline $\begin{array}{l}8 \\
9\end{array}$ & $62.0-7$ & 69 & 48.2 & - & 2.9 & 30.2 & 4.3 & - & 15.5 & - & 35 & 5. & - & - & - & $\overline{-}$ & 17 & $\overline{-}$ & - & 1 & 1.3 & - & $\overline{-}$ \\
\hline 10 & $\begin{array}{l}71.5-80 \\
805.90\end{array}$ & 79 & 25. & $z$ & - & 27.0 & 30.9 & - & 12.6 & $z$ & 33.2 & & $=$ & - & & - & & $\overline{-}$ & $\overline{-}$ & 5.8 & - & - & $\bar{z}$ \\
\hline 13 & $\begin{array}{r}809.5-90 \\
10.0-11\end{array}$ & $\begin{array}{r}89.0 \\
117.5\end{array}$ & $\begin{array}{l}20.5 \\
57.2\end{array}$ & $=$ & - & 9.1 & 30.9 & - & $=$ & - & $\begin{array}{r}7.3 \\
10.0\end{array}$ & 1.0 & 8.6 & $\overline{-}$ & $\bar{z}$ & 512 & 51.8 & - & - & $\bar{z}$ & - & $\overline{-}$ & $\overline{-}$ \\
\hline $\begin{array}{l}17 \mathrm{~A} \\
17\end{array}$ & $325.0-334.0$ & $\begin{array}{l}117.5 \\
334.0\end{array}$ & $\begin{array}{l}57.2 \\
30.3\end{array}$ & $\overline{-}$ & $\bar{z}$ & $\begin{array}{l}9.0 \\
7.4\end{array}$ & $\begin{array}{l}9.3 \\
3.6\end{array}$ & 1.8 & $\overline{4.9}$ & $\overline{-}$ & $\begin{array}{l}10.0 \\
11.0\end{array}$ & 1.8 & 8.6 & 4.0 & 11.9 & $\begin{array}{l}61.2 \\
11.7\end{array}$ & $\overline{2.3}$ & 12.9 & $\overline{-}$ & 28.0 & $\overline{-}$ & $\overline{-}$ & 0.6 \\
\hline \multicolumn{24}{|c|}{$<2 \mu \mathrm{m}$ Fractions } \\
\hline 1 & $0.0-5.0$ & 0. & 68.0 & - & - & 22. & - & - & 9.9 & 4. & 44.2 & & & - & - & - & - & - & - & - & - & - & - \\
\hline & & . & 68. & - & 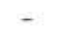 & 16 & 3.4 & 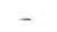 & 9.7 & 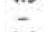 & 39.6 & 5.2 & 25 & - & - & - & - & - & - & - & - & - & - \\
\hline${ }_{4}^{3}$ & 14.5-24 & 17.0 & 69. & - & - & 19. & - & - & 8.4 & 5.6 & 41.7 & 11.5 & 13 & - & - & - & - & 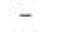 & & & & & - \\
\hline $\begin{array}{l}4 \\
5\end{array}$ & 24 & 25 & 66 & - & - & 17 & - & - & 5.0 & 3. & 43. & & & - & - & - & - & - & & & & & - \\
\hline $\begin{array}{l}5 \\
6\end{array}$ & $33.5-43$ & 41.3 & 64. & - & - & 17. & - & - & 6.1 & 3.7 & 43.9 & 10.0 & 17 & - & - & - & 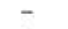 & - & 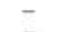 & 1.2 & & & - \\
\hline $\begin{array}{l}6 \\
8\end{array}$ & 43.0-52 & 50. & 74.9 & - & - & 22. & - & - & 8.8 & 2. & 40. & 5.3 & 18 & - & - & - & - & - & - & & & & - \\
\hline $\begin{array}{l}8 \\
9\end{array}$ & $62.0-71$ & $69 .(.2-10$ & 80.2 & - & - & 21.3 & - & - & 9.4 & 7.0 & 15.0 & 1.5 & 12 & 30. & - & - & & - & - & & & 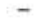 & - \\
\hline${ }_{10}^{9}$ & $\begin{array}{l}71.5-80 \\
885\end{array}$ & 79. & $\begin{array}{l}75.7 \\
72.5\end{array}$ & - & - & 13.8 & - & - & 9.4 & - & 20.6 & 2.9 & 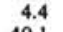 & 20.4 & - & - & 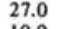 & - & & 1.5 & - & & 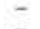 \\
\hline $\begin{array}{l}10 \\
13\end{array}$ & 80.5-90. & 89.0 & $\begin{array}{l}72.5 \\
66.7\end{array}$ & $\overline{-}$ & - & 6.2 & 13.3 & $\overline{-}$ & - & - & 5.9 & 1. & 40 & 7.8 & - & - & 10.0 & - & 14.7 & & - & & 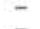 \\
\hline $17 \mathrm{~A}$ & $\begin{array}{l}109.0-118.3 \\
325.0-334.0\end{array}$ & 334.0 & $\begin{array}{l}66.1 \\
53.4\end{array}$ & $=$ & $=$ & $\begin{array}{l}4.3 \\
9.1\end{array}$ & $\overline{-}$ & 26.6 & $\overline{-}$ & $=$ & 9.6 & 2.2 & $\begin{array}{l}73.2 \\
28.2\end{array}$ & $\begin{array}{l}10.1 \\
21.1\end{array}$ & 6.2 & $\overline{-}$ & $\overline{-}$ & 1.8 & $\overline{-}$ & $\overline{7.0}$ & $=$ & $\stackrel{\mathbf{P}}{-}$ & $\overline{-}$ \\
\hline
\end{tabular}


TABLE 17

Results of X-Ray Diffraction Analysis, Site 311

\begin{tabular}{|c|c|c|c|c|c|c|c|c|c|c|c|c|c|c|c|c|c|}
\hline Core & $\begin{array}{c}\text { Cored } \\
\text { Interval } \\
\text { Below Sea } \\
\text { Floor (m) }\end{array}$ & $\begin{array}{l}\text { Sample } \\
\text { Depth } \\
\text { Below Sea } \\
\text { Floor (m) }\end{array}$ & 官 & లु & 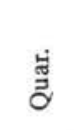 & 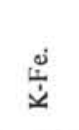 & $\frac{\text { aे }}{2}$ & $\begin{array}{l}\overrightarrow{0} \\
\ddot{g}\end{array}$ & $\stackrel{\Xi ّ}{\Sigma}$ & 열 & $\overrightarrow{\mathrm{E}}$ & 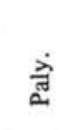 & 咅 & 氶 & 䞤 & 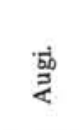 & 点 \\
\hline \multicolumn{18}{|c|}{ Bulk Samples } \\
\hline 1 & $0.0-9.0$ & $\begin{array}{l}2.5 \\
8.9\end{array}$ & $\begin{array}{l}69.1 \\
68.1\end{array}$ & $\overline{-}$ & $\begin{array}{r}38.5 \\
1.2\end{array}$ & 3.7 & $\begin{array}{l}17.0 \\
40.5\end{array}$ & - & 32.9 & $\begin{array}{c}4.2 \\
-\end{array}$ & 3.8 & - & $\overline{7} .6$ & - & - & $4 \overline{5} .5$ & $\overline{5.2}$ \\
\hline 2 & $9.0-19.5$ & $\begin{array}{l}13.1 \\
15.8\end{array}$ & 79.1 & $\overline{59.7}$ & 14.5 & $\begin{array}{l}27.6 \\
24.9\end{array}$ & $\begin{array}{l}7.0 \\
8.2\end{array}$ & - & 7.2 & - & 12.9 & - & 27.4 & - & - & - & $\begin{array}{l}3.4 \\
4.5\end{array}$ \\
\hline 4 & $22.5-28.0$ & 24.0 & 46.8 & 5.0 & - & - & - & - & - & - & 63.8 & - & 27.4 & 1.7 & - & - & 2.0 \\
\hline \multicolumn{18}{|c|}{$2-20 \mu \mathrm{m}$ Fractions } \\
\hline 1 & $0.0-9.0$ & $\begin{array}{l}2.5 \\
8.9\end{array}$ & $\begin{array}{l}43.7 \\
41.0\end{array}$ & - & $\begin{array}{r}32.9 \\
1.4\end{array}$ & 4.3 & $\begin{array}{l}16.2 \\
34.1\end{array}$ & - & 41.7 & $\begin{array}{c}4.8 \\
-\end{array}$ & - & - & $\overline{29.1}$ & - & - & $\overline{30.3}$ & $\overline{5.2}$ \\
\hline 2 & $9.0-19.5$ & $\begin{array}{l}13.1 \\
15.8\end{array}$ & $\begin{array}{l}54.8 \\
46.4\end{array}$ & - & $\begin{array}{l}9.0 \\
1.4\end{array}$ & $\begin{array}{l}32.4 \\
62.6\end{array}$ & $\begin{array}{r}9.5 \\
13.8\end{array}$ & $\overline{-}$ & $\begin{array}{r}11.2 \\
4.5\end{array}$ & - & $\overline{2.2}$ & - & $\begin{array}{r}31.7 \\
-\end{array}$ & $\overline{-}$ & - & $\begin{array}{l}- \\
-\end{array}$ & $\begin{array}{r}6.3 \\
15.6\end{array}$ \\
\hline 4 & $22.5-28.0$ & 24.0 & 18.1 & - & - & - & - & - & - & - & 65.8 & - & 26.9 & 2.8 & - & - & 4.5 \\
\hline \multicolumn{18}{|c|}{$<2 \mu \mathrm{m}$ Fractions } \\
\hline 1 & $0.0-9.0$ & 2.5 & 62.0 & - & 14.9 & 1.8 & 6.8 & 2.3 & 48.5 & 6.8 & 17.1 & - & - & - & 1.9 & - & - \\
\hline & & 8.9 & 68.2 & - & - & - & 4.6 & - & - & - & 80.5 & - & 4.2 & - & - & 9.0 & 1.7 \\
\hline 2 & $9.0-19.5$ & 13.1 & 75.4 & - & 11.7 & 16.5 & 4.8 & - & 7.0 & - & 44.7 & 6.9 & 8.5 & - & - & - & - \\
\hline & & 15.8 & 80.9 & - & 1.3 & 13.0 & 9.6 & - & 7.1 & - & 34.4 & - & 28.3 & - & - & - & 6.3 \\
\hline 4 & $22.5-28.0$ & 24.0 & 42.2 & - & - & - & - & - & - & - & 91.9 & - & 7.3 & 0.9 & - & - & - \\
\hline
\end{tabular}


TABLE 18

Results of X-Ray Diffraction Analysis, Site 313

\begin{tabular}{|c|c|c|c|c|c|c|c|c|c|c|c|c|c|c|c|c|c|c|c|c|c|c|c|c|c|}
\hline Core & $\begin{array}{l}\text { Cored } \\
\text { Interval } \\
\text { Below Sea } \\
\text { Floor (m) }\end{array}$ & $\begin{array}{l}\text { Sample } \\
\text { Depth } \\
\text { Below Sea } \\
\text { Floor (m) }\end{array}$ & 总 & లँ & 远 & 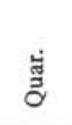 & हٌ & 安 & 递 & 宛 & 冚 & 을 & है & غं & 苞 & 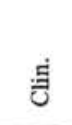 & 竎 & 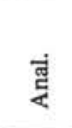 & 苞 & 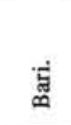 & 总 & 家 & $\begin{array}{l}\dot{\mathrm{g}} \\
\stackrel{\mathrm{J}}{\Sigma}\end{array}$ & 范 & $\frac{\pi}{5}$ \\
\hline \multicolumn{26}{|c|}{ Bulk Samples } \\
\hline 1 & $0.0-8.0$ & $\begin{array}{l}2.5 \\
6.5\end{array}$ & $\begin{array}{l}30.5 \\
65.8\end{array}$ & $\begin{array}{l}98.5 \\
46.2\end{array}$ & - & $\begin{array}{r}1.5 \\
13.5\end{array}$ & $=$ & $\overline{6} .6$ & $\overline{13.5}$ & $\overline{1.4}$ & $\overline{13.0}$ & $\overline{2.5}$ & $\overline{3.4}$ & - & - & - & $\overline{-}$ & - & $\bar{z}$ & - & - & - & - & - & 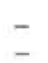 \\
\hline 2 & $35.5-45.0$ & 38.0 & 30.6 & 100.0 & - & - & - & - & - & - & - & - & - & - & - & - & - & - & - & - & - & - & - & $\overline{-}$ & - \\
\hline \multirow{2}{*}{3} & $73.5-83.0$ & 76.0 & 23.1 & 100.0 & - & - & - & - & - & - & - & - & - & - & - & - & - & - & - & - & - & - & - & - & - \\
\hline & & 80.5 & 22.9 & 100.0 & - & - & - & - & - & - & - & - & - & - & - & - & - & - & - & - & - & - & - & - & - \\
\hline 4 & $111.5-121.0$ & 118.5 & 21.1 & 100.0 & - & - & - & - & - & - & - & - & - & - & - & - & - & - & - & - & - & - & - & - & - \\
\hline 5 & $149.5-159.0$ & 151.7 & 23.1 & 100.0 & - & - & - & - & - & - & - & - & - & - & - & - & - & - & - & - & - & - & - & - & - \\
\hline 7 & $168.0-177.0$ & 174.9 & 24.2 & 100.0 & - & - & - & - & - & - & - & - & - & - & - & - & - & - & - & - & - & - & - & - & - \\
\hline 12 & $205.0-214.0$ & 207.5 & 39.5 & 100.0 & - & - & - & - & - & - & - & - & - & - & - & - & - & - & - & - & - & - & - & - & - \\
\hline 13 & $214.0-223.0$ & 216.5 & 29.0 & 100.0 & - & - & - & - & - & - & - & - & - & - & - & - & - & - & - & - & - & - & - & - & - \\
\hline 15 & $232.5-242.0$ & 234.5 & 23.7 & 98.1 & - & 0.3 & - & - & - & - & - & - & - & - & - & - & - & - & - & 1.7 & - & - & - & - & - \\
\hline 19 & $298.5-308.0$ & 305.5 & 20.8 & 100.0 & - & - & - & - & - & - & - & - & - & - & - & - & - & - & - & - & - & - & - & - & - \\
\hline 22 & $397.0-400.0$ & 399.7 & 22.9 & 98.2 & - & 0.3 & - & - & - & - & - & - & - & - & - & 1.5 & - & - & - & - & - & - & - & - & - \\
\hline 23 & $400.0-409.5$ & 409.0 & 35.0 & 87.3 & - & 1.1 & - & - & 3.7 & - & - & - & 5.7 & - & - & - & - & - & - & - & - & 2.2 & - & - & - \\
\hline \multirow{2}{*}{24} & $409.5-419.0$ & 413.3 & 39.3 & 92.4 & - & 0.6 & - & 3.6 & - & - & - & - & 3.4 & - & - & - & - & - & - & - & - & - & - & - & - \\
\hline & & 416.6 & 78.6 & 25.9 & - & 0.3 & - & 5.5 & 2.8 & - & - & - & 29.7 & - & - & - & 11.0 & 4.1 & 4.3 & - & - & 15.1 & 0.1 & 1.2 & - \\
\hline 28 & $447.5-457.0$ & 452.5 & 83.1 & 80.9 & - & 0.7 & - & 5.7 & - & - & - & - & 6.9 & - & - & - & 5.0 & - & - & - & - & - & - & 0.8 & $P$ \\
\hline 30 & $446.0-475.5$ & 466.6 & 91.2 & 68.8 & - & 0.3 & - & 13.2 & 2.5 & - & - & - & 12.9 & - & - & 1.3 & - & - & - & - & - & - & - & 1.0 & $\mathrm{~T}$ \\
\hline 31 & $475.5-485.0$ & 477.7 & 62.7 & 22.1 & 77.9 & - & - & - & - & - & - & - & - & - & - & - & - & - & - & - & - & - & - & - & - \\
\hline \multirow{3}{*}{35} & $494.5-504.0$ & 496.9 & 28.8 & 26.5 & - & 0.9 & - & 12.3 & - & - & - & - & 28.8 & - & - & - & 21.6 & 1.6 & 3.9 & - & - & - & 2.4 & 2.0 & - \\
\hline & $513.5-523.0$ & 518.8 & 27.8 & 29.0 & - & - & - & - & - & - & - & - & 67.0 & - & - & - & 3.1 & - & - & - & - & - & - & 0.9 & $\mathrm{~T}$ \\
\hline & & 520.5 & 38.3 & 88.4 & - & - & - & 2.7 & - & - & - & - & 3.9 & - & - & - & 2.8 & - & - & - & - & 2.1 & - & - & - \\
\hline 41 & $569.0-578.5$ & 576.5 & 27.3 & 90.5 & - & - & - & 3.7 & 3.7 & - & - & - & 1.3 & - & 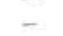 & - & - & 0.8 & - & - & - & - & - & - & $\mathrm{T}$ \\
\hline \multirow[t]{2}{*}{42} & $578.5-588.0$ & 582.8 & -21.0 & 15.2 & - & - & - & 2.9 & - & - & - & - & 76.8 & - & - & 1.7 & - & - & - & - & - & - & 0.9 & 2.4 & - \\
\hline & & 583.0 & 16.9 & 100.0 & - & - & - & - & - & - & - & - & - & - & - & - & - & - & - & - & - & - & - & - & - \\
\hline \multicolumn{26}{|c|}{ 2-20 $\mu \mathrm{m}$ Fractions } \\
\hline \multirow[t]{2}{*}{1} & $0.0-8.0$ & 2. & 56.4 & - & _ & 29.7 & - & 5. & 21 & - & 35.7 & 4.9 & - & - & - & - & - & - & - & 2.1 & - & - & - & - & - \\
\hline & & 6.5 & 42.7 & - & - & 24.9 & - & 7.4 & 27.9 & - & 33.7 & 4.0 & - & - & - & - & - & - & - & 2.1 & - & - & - & - & - \\
\hline 2 & $35.5-45.0$ & 38.0 & 80.3 & - & - & 12.8 & - & - & 35.7 & - & 7.4 & - & 10.8 & - & - & - & 16.4 & - & - & 7.9 & - & 8.9 & - & - & - \\
\hline 3 & $73.5-83.0$ & 76.0 & 82.5 & - & - & 10.2 & - & 9.1 & 11.3 & - & 4.7 & - & - & - & - & 37.7 & 27.0 & - & - & - & - & - & - & - & - \\
\hline 5 & $149.5-159.0$ & 151.7 & 87.0 & - & - & 15.2 & - & 15.6 & 26.3 & - & 6.2 & - & - & - & - & 36.6 & - & - & - & - & - & - & - & - & - \\
\hline 7 & $168.0-177.0$ & 174.9 & 49.9 & - & - & 8.8 & - & 8.5 & 18.8 & - & 11.5 & 1.8 & 14.9 & - & - & 33.8 & - & - & - & 1.9 & - & - & - & - & - \\
\hline 12 & $205.0-214.0$ & 207.5 & 100.0 & - & - & - & - & - & - & - & - & - & - & - & - & - & - & - & - & - & - & - & - & - & - \\
\hline 13 & $214.0-223.0$ & 216.5 & 99.4 & - & - & 14.7 & - & 55.4 & - & - & - & - & - & - & - & - & - & - & - & 29.9 & - & - & - & - & - \\
\hline 15 & $232.5-242.0$ & 234.5 & 37.9 & - & - & 14.8 & - & 3.1 & 11.4 & - & 6.1 & - & - & - & - & 29.1 & - & - & - & 35.5 & - & - & - & - & - \\
\hline 19 & $298.5-308.0$ & 305.5 & 44.3 & - & - & 8.0 & - & - & 8.5 & - & 6.7 & - & 9.5 & - & - & 67.3 & - & - & - & - & - & - & - & - & - \\
\hline 22 & $397.0-400.0$ & 399.7 & 73.4 & - & - & 5.1 & 10.2 & 8.2 & 6.2 & - & 5.5 & - & 7.6 & - & 4.1 & 47.3 & - & - & - & 5.9 & - & - & - & - & - \\
\hline 23 & $400.0-409.5$ & 409.0 & 49.8 & - & - & 0.5 & - & ${ }_{-}^{0.2}$ & 7.4 & - & - & - & 25.3 & - & - & - & - & - & - & - & - & 66.8 & - & - & - \\
\hline \multirow[t]{2}{*}{24} & $409.5-419.0$ & 413.3 & 47.5 & - & - & 4.6 & - & 35.0 & 9.1 & - & 12.4 & - & 8.2 & - & - & 3.0 & - & - & 14.8 & 3.1 & - & 4.7 & 4.5 & 0.5 & - \\
\hline & & 416.6 & 44.7 & - & - & - & - & 4.8 & 4.8 & - & 1.9 & - & 20.4 & - & - & - & 31.4 & 10.6 & 5.5 & - & - & 11.2 & 7.3 & 2.2 & - \\
\hline 28 & $447.5-457.0$ & 452.5 & 36.2 & - & - & 2.4 & - & 21.4 & 5.5 & - & 2.3 & - & 19.6 & - & - & - & 40.0 & - & 2.2 & - & 2.5 & - & 2.8 & 1.3 & - \\
\hline 30 & $466.0-475.5$ & 466.6 & 34.5 & - & - & 3.1 & - & 66.6 & 14.5 & - & 5.0 & 0.4 & 3.8 & - & - & - & - & - & 4.6 & - & 2.0 & .- & - & - & - \\
\hline 31 & $475.5-485.0$ & 477.7 & 47.4 & - & - & 3.1 & - & 32.7 & 6.3 & - & 3.5 & - & 3.7 & - & - & 41.9 & - & 0.8 & 2.8 & 3.0 & - & - & 2.3 & - & - \\
\hline 33 & $494.5-504.0$ & 496.9 & 46.1 & - & - & 2.4 & - & 21.6 & - & - & - & - & 34.5 & - & - & - & 25.7 & 2.7 & 2.1 & - & 0.7 & - & 6.6 & 3.7 & - \\
\hline \multirow{2}{*}{35} & $513.5-523.0$ & 518.8 & 37.6 & - & - & 0.8 & - & 10.4 & 3.0 & - & - & - & 64.7 & - & - & - & 13.9 & - & 0.9 & - & - & - & 2.4 & 4.0 & - \\
\hline & & 520.5 & 84.9 & - & - & 4.0 & - & 26.2 & 6.0 & - & 5.8 & - & 11.3 & - & - & 0.7 & 19.8 & 0.6 & 5.4 & - & 0.6 & 11.5 & 6.2 & 1.9 & - \\
\hline 41 & $569.0-578.5$ & 576.5 & 72.5 & - & - & 1.7 & - & 47.9 & 20.8 & - & 12.1 & - & 6.4 & - & - & - & - & 4.9 & - & - & 2.5 & - & 3.6 & - & - \\
\hline \multirow[t]{2}{*}{42} & $578.5-588.0$ & 582.8 & 48.7 & - & - & 0.8 & - & 15.3 & - & - & - & - & 66.1 & - & - & 1.6 & - & - & - & - & - & - & 11.3 & 5.0 & - \\
\hline & & 583.0 & 99.4 & - & - & 7.0 & - & 5.7 & 5.0 & - & - & - & - & - & - & 64.0 & - & - & - & 18.4 & - & - & - & - & 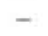 \\
\hline
\end{tabular}


$<2 \mu \mathrm{m}$ Fractions

\begin{tabular}{|c|c|c|c|c|c|c|c|c|c|c|c|c|c|c|c|c|c|c|c|c|c|c|c|c|c|}
\hline 1 & $0.0-8.0$ & 2.5 & 69.7 & - & - & 15.5 & - & - & 8.5 & . & 39.1 & 8.1 & 8.4 & - & - & - & 20.4 & - & - & - & - & - & - & - & - \\
\hline & & 6.5 & 75.7 & - & - & 18.3 & - & 5.7 & 18.5 & 2.5 & 30.7 & 3.9 & 20.3 & & & - & - & - & - & - & - & - & - & - & - \\
\hline \multirow{4}{*}{3} & $35.5-45.0$ & 38.0 & 75.7 & - & - & 9.2 & - & & 13.7 & - & 11.4 & 2.9 & 47.1 & & - & - & 6.0 & - & - & 3.3 & - & 6.4 & - & - & - \\
\hline & $73.5-83.0$ & 76.0 & 85.7 & - & - & 9.7 & - & 3.7 & 9.6 & - & 12.0 & 2.7 & 27.1 & & & 20.1 & 15.1 & - & - & - & - & - & - & - & - \\
\hline & & 80.5 & 82.2 & - & - & 2.8 & - & 4.7 & 7.7 & & 6.6 & $\because$ & 41.3 & & & 18.2 & 18.7 & - & - & - & - & - & - & - & - \\
\hline & $111.5-121.0$ & 118.5 & 97.6 & - & - & 5.8 & - & 10.4 & 9.0 & - & 14.2 & - & 26.3 & - & - & 20.2 & 14.2 & - & - & - & - & - & - & - & - \\
\hline 5 & $149.5-159.0$ & 151.7 & 58.6 & - & - & 3.9 & - & 4.4 & 7.8 & 4.9 & 7.7 & 2.5 & 59.6 & - & & 9.2 & - & - & - & - & - & - & - & - & - \\
\hline 7 & $168.0-177.0$ & 174.9 & 74.9 & - & - & 4.4 & - & 6.0 & 8.5 & 12.0 & 9.1 & - & 52.4 & & - & 6.7 & - & - & - & 1.0 & - & - & - & - & - \\
\hline 12 & $205.0-214.0$ & 207.5 & 92.7 & - & _- & 7.2 & - & 10.3 & 10.7 & - & $\ldots$ & 5.5 & 63.3 & $\ldots$ & - & - & - & - & - & 3.0 & - & - & - & - & - \\
\hline 13 & $214.0-223.0$ & 216.5 & 93.7 & - & - & 5.9 & - & 13.4 & - & 4.3 & - & - & 73.2 & -. & - & - & - & - & - & 3.2 & - & - & - & - & - \\
\hline 15 & $232.5-242.0$ & 234.5 & 71.3 & - & - & 11.2 & - & 2.4 & 4.6 & 1.1 & 9.7 & & 37.6 & 21.0 & & 7.1 & & _- & - & 5.4 & - & - & - & - & - \\
\hline 19 & $298.5-308.0$ & 305.5 & 70.5 & - & - & 8.1 & - & 3.3 & 2.8 & 2.2 & 10.4 & & 55.5 & 8.0 & - & 7.7 & - & - & - & 1.9 & - & - & - & - & - \\
\hline 22 & $397.0-400.0$ & 399.7 & 74.2 & - & - & 2.6 & 27.8 & 2.9 & 2.9 & - & 6.4 & & 40.5 & 10.6 & 3.2 & - & - & - & - & 3.3 & - & - & - & - & - \\
\hline 23 & $400.0-409.5$ & 409.0 & 39.5 & - & - & 0.4 & - & & 2.4 & - & & - & 89.9 & - & - & - & _- & - & - & - & - & 7.3 & - & - & - \\
\hline \multirow[t]{2}{*}{24} & $409.5-419.0$ & 413.3 & 66.6 & - & - & 2.0 & - & 7.8 & - & - & 8.4 & - & 72.5 & - & - & - & - & - & 4.9 & 1.6 & - & 1.1 & - & 1.8 & - \\
\hline & & 416.6 & 49.2 & - & - & - & - & - & 1.6 & - & 2.3 & - & 86.9 & - & - & - & 5.9 & 1.2 & - & - & - & - & - & 2.2 & - \\
\hline 28 & $447.5-457.0$ & 452.5 & 57.2 & - & - & - & - & - & - & - & 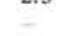 & & 94.6 & _- & - & - & 3.0 & - & - & - & - & - & - & 2.4 & - \\
\hline 30 & $466.0-475.5$ & 466.6 & 61.5 & - & - & 0.9 & - & 6.2 & 2.6 & & - & - & 85.3 & - & - & - & - & - & 2.9 & - & - & - & - & 2.0 & - \\
\hline 31 & $475.5-485.0$ & 477.7 & 58.8 & - & - & 1.3 & - & - & - & & - & & 94.4 & - & - & 1.8 & - & - & - & 1.5 & - & - & - & 1.1 & - \\
\hline 33 & $494.5-504.0$ & 496.9 & 43.3 & - & - & - & - & 1.6 & - & - & - & - & 83.4 & - & - & - & 8.8 & 0.7 & - & - & - & - & 2.5 & 3.0 & - \\
\hline \multirow[t]{2}{*}{35} & $513.5-523.0$ & 518.8 & 31.1 & - & - & - & - & - & - & - & - & - & 99.3 & - & - & - & - & - & - & - & - & - & - & 0.7 & - \\
\hline & & 520.5 & 59.7 & - & - & 1.0 & - & 2.9 & 1.9 & - & 4.3 & - & 85.6 & - & - & - & - & 0.6 & 1.5 & - & - & - & - & 2.3 & - \\
\hline 41 & $569.0-578.5$ & 576.5 & 77.5 & - & - & 1.9 & - & 10.2 & 8.3 & - & - & - & 71.8 & - & - & - & - & 2.1 & 3.9 & - & - & - & - & 1.7 & - \\
\hline \multirow[t]{2}{*}{$\begin{array}{l}41 \\
42\end{array}$} & $578.5-588.0$ & 582.8 & 51.3 & - & - & - & - & - & - & - & - & - & 96.4 & - & - & - & - & - & - & - & - & - & - & 3.6 & - \\
\hline & & 583.0 & 81.7 & - & - & 10.2 & - & - & - & - & - & - & 72.7 & - & - & 7.1 & - & - & - & 10.0 & - & - & - & - & - \\
\hline
\end{tabular}

${ }^{\mathrm{a}} \mathrm{U}-1$ identifiable peak located at $3.07 \AA$. 